Draft VERSION SEPTEMBER 24, 2018

Preprint typeset using $\mathrm{LAT}_{\mathrm{E}} \mathrm{X}$ style AASTeX6 v. 1.0

\title{
OBSERVATIONAL EVIDENCE OF DYNAMIC STAR FORMATION RATE IN MILKY WAY GIANT MOLECULAR CLOUDS
}

\author{
Eve J. LeE ${ }^{1}$, Marc-Antoine Miville-Deschênes ${ }^{2}$, Norman W. Murray ${ }^{3,4}$
}

\footnotetext{
${ }^{1}$ Astronomy Department, University of California, Berkeley, CA 94720, USA; evelee@berkeley.edu

${ }^{2}$ Institut d'Astrophysique Spatiale, CNRS/Université Paris-Sud 11, 91405 Orsay, France

${ }^{3}$ Canadian Institute for Theoretical Astrophysics, 60 St. George Street, University of Toronto, Toronto ON M5S 3H8, Canada

${ }^{4}$ Canada Research Chair in Theoretical Astrophysics
}

\section{ABSTRACT}

Star formation on galactic scales is known to be a slow process, but whether it is slow on smaller scales is uncertain. We cross-correlate 5469 giant molecular clouds (GMCs) from a new all-sky catalog with 256 star forming complexes (SFCs) to build a sample of 191 SFC-GMC complexes - collections of multiple clouds each matched to 191 SFCs. The total mass in stars harbored by these clouds is inferred from WMAP free-free fluxes. We measure the GMC mass, the virial parameter, the star formation efficiency $\epsilon$ and the star formation rate per free-fall time $\epsilon_{\mathrm{ff}}$. Both $\epsilon$ and $\epsilon_{\mathrm{ff}}$ range over $3-4$ orders of magnitude. We find that $68.3 \%$ of the clouds fall within $\sigma_{\log \epsilon}=0.79 \pm 0.22$ dex and $\sigma_{\log \epsilon_{\mathrm{ff}}}=0.91 \pm 0.22 \mathrm{dex}$ about the median. Compared to these observed scatters, a simple model with a time independent $\epsilon_{\mathrm{ff}}$ that depends on the host GMC properties predicts $\sigma_{\log \epsilon_{\mathrm{ff}}}=0.12-0.24$. Allowing for a time-variable $\epsilon_{\mathrm{ff}}$, we can recover the large dispersion in the rate of star formation. This strongly suggests that star formation in the Milky Way is a dynamic process on GMC scales. We also show that the surface star formation rate profile of the Milky Way correlates well with the molecular gas surface density profile.

\section{INTRODUCTION}

Star formation is a slow process on galactic size and time scales, with a mere $\sim 2 \%$ of the gas mass turning into stars in the disk dynamical time (Kennicutt 1989, 1998). Stars in the Milky Way and nearby galaxies form in giant molecular clouds (GMCs), with the mass in newly formed stars proportional to the mass in host GMCs (see e.g., Mooney \& Solomon 1988; Scoville \& Good 1989). The bulk of the molecular gas resides in the most massive GMCs (e.g., Solomon et al. 1987) and, as implied by the results of Mooney \& Solomon (1988), most star formation occurs in the most massive GMCs (see also e.g., Murray 2011).

There is considerable disagreement regarding the rate of star formation and the star formation efficiencies on scales of GMCs and smaller. Star formation efficiency $\epsilon$ is defined as the ratio of the mass in protostars to the total mass in a given star-forming region:

$$
\epsilon \equiv \frac{M_{\star}}{M_{g}+M_{\star}},
$$

where the star-forming region may be a GMC or a smaller sub-region of a GMC. The star formation rate per free fall time $\epsilon_{\mathrm{ff}}$ is

$$
\epsilon_{\mathrm{ff}} \equiv \epsilon \frac{\tau_{\mathrm{ff}}}{\tau_{\star}},
$$

where $\tau_{*}$ is the lifetime of the (proto-)stellar object in question, and $\tau_{\mathrm{ff}} \equiv \sqrt{3 \pi / 32 G \rho}$ is the free fall time of the star-forming region (a GMC or its sub-region), which is assumed to have a mean density $\rho$.

Mooney \& Solomon (1988) and Scoville \& Good (1989) showed that there is a wide (maximum to minimum of approximately $2.5 \mathrm{dex}$ ) spread in the efficiencies of star formation in GMCs, a measurement based on the ratio of far infrared luminosity $L_{\text {FIR }}$ to CO luminosity $L_{\mathrm{CO}}$. More recent estimates also employ counts of protostars in nearby Milky Way molecular clouds (e.g. Evans et al. 2009; Heiderman et al. 2010; Lada et al. 2010), or measurements of the free-free emission associated with massive stars (Murray 2011) to find a similarly large spread. Heiderman et al. (2010) and Lada et al. (2010) (as well as Evans et al. 2009 to a smaller degree) find broad distributions of $\epsilon_{\mathrm{ff}}$, which range both well below and well above $\epsilon_{\mathrm{ff}}=0.02$, by factors of $\sim 20$ or more in either direction; they also note that Galactic clouds with high surface densities may have higher-than-expected $\epsilon_{\mathrm{ff}}$ compared to their extragalactic counterparts.

Krumholz et al. (2012) argue that the broad distribution in $\epsilon_{\mathrm{ff}}$ of Galactic molecular clouds can be explained by variations in volumetric densities (the density determining the free-fall time) among clouds. They 
argue that the star formation rate on all scales is $\epsilon_{\mathrm{ff}} \approx$ $0.02 M_{\mathrm{GMC}} / \tau_{\mathrm{ff}}$, with only a factor of 3 scatter above and below the mean value, once the variations in $\tau_{\mathrm{ff}}$ are taken into account (see also e.g., Krumholz \& Tan 2007).

However, using a sample of clouds from c $2 \mathrm{~d}$ and Gould Belt Spitzer legacy programs, Evans et al. (2014) find a large scatter in $\epsilon_{\mathrm{ff}}$ even after taking into account the variations in volumetric densities. Similarly, Heyer et al. (2016) study dense clumps in ATLASGAL survey and find $\epsilon_{\mathrm{ff}} \sim 0.001-0.01$ (see their Figure 10c).

The rate of small scale star formation, and the dispersion of its distribution, is important for galaxy formation. If the small scale star formation rate is steady and hence low, the mass in live stars will be steady, and small. ${ }^{1}$ It follows that the kinetic and thermal feedback from stellar winds, radiation, and supernovae will be steady and low. If the small scale star formation is sporadic, with pronounced peaks and long-lived lows, the feedback will be both temporally and spatially concentrated. Because stars form preferentially in dense gas, the resultant feedback will be deposited in regions of dense gas, where it has the potential to move the most material, but also where cooling is most rapid. Observations of starburst galaxies show that massive star clusters are prominent sources of galaxy scale winds (e.g., Schwartz et al. 2006). These winds are believed to be crucial for determining both the global star formation rate and the total stellar mass by regulating the amount of gas in the disk (e.g., Oppenheimer et al. 2010; Hopkins et al. 2011, 2014), as well as the distribution of metals in the intergalactic medium (e.g., Oppenheimer et al. 2010). For a fixed global star formation rate, sporadic small scale star formation will tend to produce more massive clusters than will steady small scale star formation, with important consequences for wind properties.

Star formation is promoted by gravity and by convergent fluid flows, and suppressed by a number of physical effects including thermal gas pressure, turbulent kinetic pressure, magnetic fields, and stellar feedback i.e. stellar winds, radiation pressure, protostellar jets, and, at late times, supernovae.

Two leading candidates (at the time of writing) for the suppression of star formation rates are stellar feedback and turbulent pressure support. On galactic scales, the rate of star formation is believed to be regulated by stellar feedback, which can keep the gas disk in a state of marginal stability (e.g., Thompson et al. 2005; Ostriker \& Shetty 2011). The turbulent pressure scenario

\footnotetext{
1 We refer to stars less than 4 Myrs old as "young" or "live" stars. Clusters containing more than $\sim 5000 M_{\odot}-10000 M_{\odot}$, which sample the initial mass function (IMF) fairly well, have ionizing photon rates $Q\left(\mathrm{~s}^{-1}\right)$ that are dominated by massive stars with $M_{\star} \gtrsim 30 M_{\odot}$. Such stars have lifetimes of order 4 Myrs.
}

is well motivated: the large linewidths seen in massive star forming regions (e.g., Caselli \& Myers 1995; Plume et al. 1997) show that the kinetic energy density greatly exceeds the thermal pressure on scales larger than $\sim 0.01 \mathrm{pc}$, and is comparable to the gravitational potential energy density. ${ }^{2}$ A number of authors have suggested that these turbulent motions support GMCs and hence slow the rate of star formation. The extreme version of the argument says that turbulence maintains GMCs in hydrostatic equilibrium, preventing large scale collapse (e.g., Myers \& Fuller 1992; McLaughlin \& Pudritz 1997; McKee \& Tan 2003).

If GMCs are in hydrostatic equilibrium (which implies that the clouds live for at least a few free fall times) then $\epsilon_{\mathrm{ff}}=0.02$ implies that the mass in live stars is roughly the same in most GMCs of a given mass. However, recent numerical and semi-analytic studies show that $\epsilon_{\mathrm{ff}}$ increases roughly linearly with time (e.g., Lee et al. 2015; Murray \& Chang 2015; Murray et al. 2015). If this is true, then most $10^{6} M_{\odot}$ GMCs will have very few live stars, while a small subset of $10^{6} M_{\odot}$ GMCs will host very massive clusters of live stars; the distribution of $\epsilon_{\mathrm{ff}}$ will be very broad.

Measuring the width of the distribution in $\epsilon_{\mathrm{ff}}$ and $\epsilon$ therefore provides an important diagnostic for testing the idea that $\epsilon_{\mathrm{ff}}$ is independent of time. We will show that the observed scatter in $\epsilon_{\mathrm{ff}}$ of the Milky Way GMCs is significantly larger than what is predicted by models of constant star formation rate per free fall time (e.g., Krumholz \& McKee 2005; Padoan et al. 2012).

To estimate either $\epsilon$ or $\epsilon_{\mathrm{ff}}$ we must estimate the mass in young stars. We use the free-free flux to do so. Our sample of star-forming Milky Way GMCs is built by cross-correlating a new all-sky cloud catalog from M-A., Miville-Deschênes et al. (2016, in preparation; MML16 from hereon) with star forming complexes (SFCs) from Lee et al. (2012).

This paper is organized as follows: in Section 2, we match SFCs with GMCs in (l,b,v)-space (galactic longitude, galactic latitude, radial velocity); in Section 3, we describe how we convert free-free flux into stellar mass; in Sections 4 and 5, we present our analysis of $\epsilon$ and $\epsilon_{\mathrm{ff}}$, respectively; in Section 6, we compare various models of star formation rate to observations; in Section 7, we present the surface density star formation rate profile across the Galactic plane; we summarize and discuss our results in Section 8.

\footnotetext{
2 The two pictures - stellar feedback and turbulence - are not necessarily in conflict, since a source of energy is needed to power the turbulence seen in GMCs, and stellar feedback may provide this energy.
} 


\section{STAR FORMING GIANT MOLECULAR CLOUDS}

In this section, we describe how we cross-correlate the SFCs in Lee et al. (2012) with the GMC catalog of MML16.

\subsection{Star Forming Complexes}

We present a brief description of the SFC catalog here (see Lee et al. 2012, for more detail). Lee et al. (2012), following the approach of Murray \& Rahman (2010) and Rahman \& Murray (2010), identified 280 SFCs in and near the Galactic plane.

Ionizing photons from young clusters can travel tens or even hundreds of parsecs through the interstellar medium before being absorbed. It follows, and it is observed, that free-free emission regions can be much larger $\left(\sim 1-2^{\circ}\right.$ in diameter $)$ than the star clusters that power them. In addition, star clusters tend to form in associations (just like stars tend to form in clusters), so that the free-free emission seen in a given direction may be powered by more than one star cluster. Star forming complexes represent systems of clusters that are able to carve out bubbles of size $\sim 10-100$ parsecs in their host GMCs. We assign a free-free flux $f_{\nu}$ to each SFC by dividing up the free-free flux of their host WMAP sources (the $1-2^{o}$ wide regions of free-free emission) in proportion to the $8 \mu \mathrm{m}$ flux of each SFC, as seen by Spitzer. This is motivated by the linear correlation between freefree and $8 \mu \mathrm{m}$ flux seen on small (parsec) scales.

Lee et al. (2012) calculated the distance to each SFC by fitting the galactic longitude and the central local standard at rest velocity $v_{\text {lsr }}$ to the Clemens (1985, hereafter C85) rotation curve. For SFCs that reside inside the solar circle $\left(R_{\odot} \leq 8.5 \mathrm{kpc}\right)$, they use the radial velocities of the absorption lines along the line of sight to disambiguate between near (absorption line velocity is less than that of the SFC) and far (absorption line velocity can be as large as the tangent point velocity) distances.

\subsection{Rotation Curve and Kinematic Distance}

The rotation curve calculated by C85 is known to introduce significant errors in the distance to the objects in the outer galaxy due to Perseus arm streaming motions (Fich et al. 1989). We therefore update the Lee et al. (2012) distances using the rotation curve of Brand \& Blitz (1993, hereafter BB93) at $R_{0}=8.5 \mathrm{kpc}$ and $\Theta_{0}=220 \mathrm{~km} \mathrm{~s}^{-1}$.

Figure 1 shows that the distances given by the two rotation curves are generally in good agreement. The four outliers with C85 'near' distances that are close to zero have $\mathrm{C} 85$ solutions that are slightly inside (by $\sim 0.03 \mathrm{kpc}$ ) the solar circle, while the BB93 solutions

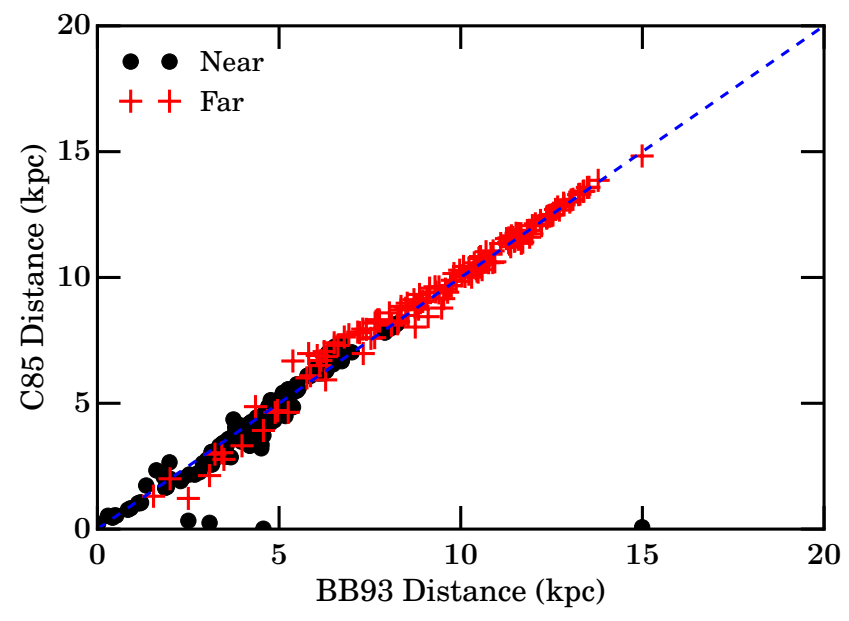

Figure 1. Comparison of the kinematic distances to SFCs inferred from using the Clemens (1985) and Brand \& Blitz (1993) rotation curves. Black circles represent near distances, while red crosses represent far distances. The blue dashed line shows the relationship that would be found if the two curves gave the same distances. For the four outliers, where the Clemens (1985) curve predicts near-zero distances, the Brand \& Blitz (1993) curve predicts a unique large distance. The Clemens (1985) curve yields a galactocentric radius that is slightly inside the solar circle, while the Brand \& Blitz (1993) rotation curve finds a galactocentric radius that is slightly outside the solar circle.

for these objects are slightly outside (by $\sim 0.12 \mathrm{kpc}$ ) the solar circle.

We reject solutions with galactocentric radius greater than $16 \mathrm{kpc}$, i.e., we are using a prior that there is no significant massive (O star) star formation at such large radii. The typical error in distance is $\sim 35 \%$, the dominant sources of error being the streaming velocity of ionized gas, the expansion velocities of bubbles carved out by SFCs, and the motions of spiral arms (Lee et al. 2012).

\subsection{Giant Molecular Clouds}

We use the new all-sky catlaog of Galactic molecular clouds presented by MML16, where we describe in detail how clouds are identified. MML16 present a number of correlations between cloud properties. We provide a brief summary here.

Clouds are identified as coherent molecular structures from the ${ }^{12} \mathrm{CO}$ (J1-0) survey of Dame et al. (2001). This survey has a modest angular resolution ( $7.5 \mathrm{arcmin})$ compared to other observations, e.g., Jackson et al. (2006), but it has the advantage of providing a uniform data set covering the entire Galactic plane in longitude. We limit our study to $-5^{\circ}<b<5^{\circ}$.

The identification of clouds from position-positionvelocity (or $[l, b, v]$ ) cubes is challenging, as the interstellar medium (ISM) has a fractal structure, and because unrelated clouds along a given line of sight can have sim- 
ilar projected velocities. Given this difficulty, it is striking that studies using different data sets and different structure identification techniques find relatively consistent scaling relations between various cloud properties, such as size, velocity dispersion, mass and surface density (see the review by Hennebelle \& Falgarone 2012). MML16 find scaling relations similar to those reported in the earlier catalogs.

MML16 employ a combination of Gaussian decomposition and hierarchical clustering analysis to identify clouds. First, every spectra of the the entire CO position-position-velocity (PPV) cube is decomposed into a sum of Gaussian components. Next, they build a cube of integrated emission $W_{\mathrm{CO}}$ where each grid cell $(l, b, v)$ is assigned $W_{\mathrm{CO}}$ integrated over Gaussian components whose central velocities equal $v$. This cube is much more sparse than the original brightness cube as the integrated emission of each Gaussian component is concentrated in a single cell $(l, b, v)$ and not spread out in velocity. This new cube facilitates the identification of coherent structures down to the noise level of the data. To do so, they used a classical threshold descent. First, they identified islands in PPV space as neighboring cells with $W_{\mathrm{CO}}$ higher than some high threshold value. The threshold is progressively lowered down to $0.5 \mathrm{~K} \mathrm{~km} \mathrm{~s}^{-1}$. At each step of this descent, new cells are revealed. They are either attached to previously identified structures or classified as new structures. This watershed method is similar in spirit to clumpfind (Williams et al. 1994) or to dendrogrammes (Rosolowsky et al. 2008). A total of 8107 coherent structures / clouds are identified over the whole Milky Way disk, recovering $91 \%$ of the total CO emission.

As for the SFCs in this paper, the distance to each GMC is estimated assuming that its average velocity follows the Galactic rotation curve of Brand \& Blitz (1993). In the inner Galaxy, there is an ambiguity as two distances along the line of sight (dubbed near and far) have the same observed velocity. MML16 relied on a statistical method that has been used by many other studies (e.g., Dame et al. 1986; Solomon et al. 1987; Grabelsky et al. 1988; García et al. 2014). The original idea was to choose the distance that provides a cloud physical size, $R$, that matches more closely the size-linewidth $\sigma_{v} \propto R^{\alpha}$ relation, established using clouds not subject to the distance ambiguity.

The size-linewidth relation appears to vary with the cloud column density $\Sigma$ (see e.g., Heyer et al. 2009). To account for this dependence on $\Sigma$, MML16 select the distance that best matches the relation $\sigma \propto(R \Sigma)^{0.42}$. The cloud distances chosen in this manner agree well with the distances of associated SFCs, as we show in Section 2.4.

The cloud catalog provides the position in $(l, b, v)$, the size, the velocity dispersion, and the distance to each cloud. It also provides the gas mass estimated as

$$
M_{\mathrm{g}}=W_{\mathrm{CO}}^{\mathrm{tot}} X_{\mathrm{CO}} 2 \mu m_{\mathrm{H}} D^{2} \tan (\delta)^{2}
$$

where $W_{\mathrm{CO}}^{\text {tot }}$ is the total CO emission of all the Gaussian components associated to the cloud, $X_{\mathrm{CO}}=2 \times$ $10^{20} \mathrm{~cm}^{-2} \mathrm{~K}^{-1} \mathrm{~km}^{-1} \mathrm{~s}$ (Bolatto et al. 2013), $\mu=1.36$ takes into account the contribution fron Helium, $m_{\mathrm{H}}$ is the mass of Hydrogen, $D$ is the distance to the cloud and $\delta=0.125^{\circ}$ is the angular size of the pixel. We also provide the WMAP free-free and IRAS $100 \mu \mathrm{m}$ flux of clouds, measured by integrating the emission over pixels associated with each cloud.

\subsection{Cross-Correlating Star Forming Complexes with Giant Molecular Clouds}

We identify matches between SFCs and GMCs if they meet the following criteria:

$$
\sqrt{\left(\delta l^{2}+\delta b^{2}+\delta v^{2}\right)} \leq 1
$$

where

$$
\begin{aligned}
\delta l & =\left(l_{G M C}-l_{S F C}\right) / \max \left(\sigma_{\mathrm{l}, \mathrm{GMC}}, \mathrm{R}_{\mathrm{SFC}} / \mathrm{D}, 0.5^{\circ}\right) \\
\delta b & =\left(b_{G M C}-b_{S F C}\right) / \max \left(\sigma_{\mathrm{b}, \mathrm{GMC}}, \mathrm{R}_{\mathrm{SFC}} / \mathrm{D}, 0.5^{\mathrm{o}}\right) \\
\delta v & =\left(v_{G M C}-v_{S F C}\right) / \max \left(\sigma_{\mathrm{v}, \mathrm{GMC}}, \sigma_{\mathrm{v}, \mathrm{SFC}}, 7 \mathrm{~km} \mathrm{~s}^{-1}\right) .
\end{aligned}
$$

By $R_{S F C} / D$ we mean the angular size of the SFC measured in degrees rather than radians; $\sigma_{v, \mathrm{GMC}}$ is the RMS velocity dispersion of a GMC while $\sigma_{v, \mathrm{SFC}}$ is the halfspread velocity of SFCs (i.e., $\left(v_{\max }-v_{\min }\right) / 2$ along the bubble walls; see Lee et al. 2012 for more detail). Out of $280 \mathrm{SFCs}, 256$ have measured median velocities $v_{\mathrm{SFC}}$. Since the host $W M A P$ sources are $\sim 2^{\circ}$ in size, we only allow SFC-GMC matches to the clouds that are large enough to encompass more than 10 pixels but smaller than $2^{o}$ in their longest axis; larger objects are likely not isolated, self-gravitating clouds. ${ }^{3}$ The selection criteria on the GMC size limits the cloud count to 5469 . In summary, we perform a cross-correlation between 256 SFCs and 5469 GMCs.

Using the criteria given by equations 4 and 5 , we find that approximately half of SFCs are matched with multiple GMCs. Visual inspection reveals that these cloudswhich are often smaller in size than SFCs - trace the outer rim of bubbles blown by their SFC counterpart when we overlay them on GLIMPSE $8 \mu \mathrm{m}$ images. It

\footnotetext{
${ }^{3}$ We note that there are a couple of SFCs with mean radii that are close to or exceed $2^{\circ}$, such as SFC Nos. 110 and 111. These SFCs are associated with the Cygnus region within a particularly large $W M A P$ free-free source (it was originally identified as three separate free-free sources by Murray \& Rahman (2010) but Lee et al. (2012) merged them after a visual inspection).
} 
is likely that the clouds originate from a single massive cloud that was disrupted by stellar winds and radiation pressure from its massive star clusters. These SFC-GMC "complexes" are often found inside the solar circle, so that even though multiple objects (SFCs or GMCs) coincide in the $(l, b, v)$-space, they can be at vastly different distances. We reject any GMC that is not within $0.3 d_{\mathrm{SFC}}$ from the centroid of the host SFC, where $d_{\mathrm{SFC}}$ is the heliocentric distance of the SFC. For SFCs without a distance measurement, we use the distance of the GMC that is most closely matched in the $(l, b, v)$-space.

Lastly, we make a visual inspection for the luminous SFCs (those with expected $M_{\star} \geq 10^{4} M_{\odot}$ ) to ensure that we have made a sensible cross-correlation. Only $\sim 10 \%$ of the initial SFC-GMC complexes are mismatched. Most of the mismatches stem from conflicting distance ambiguity resolution between the SFC catalog and the GMC catalog.

The fact that most of the luminous SFCs are matched with GMCs suggests a good agreement in distance ambiguity resolution between the SFC sample (resolution by absorption line velocities) and the GMC sample (resolution by a fit to a size-linewidth-column-density relation), enhancing our confidence in the use of the latter technique.

We identify a known cluster from Morales et al. (2013) in the direction of SFC-GMC complexes to reassign SFC Nos. 27, 28, 93 to near distances and GMC Nos. 136, $171,388,398,446,523,625,812,1312,1656,2733,2734$, 2816, 3422 to far distances. ${ }^{4}$ Distances to SFC Nos. 36 and 252 are changed to far distances after correcting for a bug in a code used to produce the catalog in Lee et al. (2012). We also manually match SFC No. 202 with GMC No. 2420, SFC No. 65 with GMC No. 2071 (associated with the W40 cluster (Mallick et al. 2013)), and SFC No. 251 with GMC No. 482 (associated with RCW 120 (Anderson et al. 2010)). Four SFCs (Nos. $31,35,245,248)$ had no GMC match because their distances were truncated to $12 \mathrm{kpc}$ (because of their likely association with $\sim 3 \mathrm{kpc}$ ring; see Lee et al. 2012); we recalculated their distances.

We present the results of our cross-correlation in Table 1 .

The final result is 191 unique SFCs matched to 389 unique GMCs, recovering $93.5 \%$ of the total SFC freefree luminosity, $83.8 \%$ of the total SFC free-free flux, and $9 \%$ of total GMC gas mass. All of the top 24 most lu-

${ }^{4}$ SFC Nos. 27 and 28 are associated with the W31 cluster while SFC No. 93 is associated with [BDS2003] 135 (Bica et al. 2003). minous SFCs are matched to at least one GMC. For the rest of the paper, we identify these 389 GMCs matched to 191 SFCs as 191 SFC-GMC complexes.

Each complex inherits the sum of the $\mathrm{CO}$ fluxes and gas masses of the matched GMCs, as well as the massweighted mean Galactic coordinates $l, b$, velocity $v$, heliocentric distance $d$, and galactocentric radius $R_{\text {gal }}$. Following MML16, we calculate the angular size and surface area of each SFC-GMC complex by solving for eigenvalues of the moment of inertia matrix:

$$
\phi=\left[\begin{array}{cc}
\sigma_{l}^{2} & \sigma_{l b}^{2} \\
\sigma_{l b}^{2} & \sigma_{b}^{2}
\end{array}\right]
$$

where

$$
\begin{aligned}
\sigma_{l}^{2} & =\frac{\sum_{i} M_{i} \sum_{c=1}^{4}\left(l_{c, i}-l_{\mathrm{SG}}\right)^{2}}{4 \sum_{i} M_{i}} \\
\sigma_{b}^{2} & =\frac{\sum_{i} M_{i} \sum_{c=1}^{4}\left(b_{c, i}-b_{\mathrm{SG}}\right)^{2}}{4 \sum_{i} M_{i}} \\
\sigma_{l b}^{2} & =\frac{\sum_{i} M_{i} \sum_{c=1}^{4}\left(l_{c, i}-l_{\mathrm{SG}}\right)\left(b_{c, i}-b_{\mathrm{SG}}\right)}{4 \sum_{i} M_{i}} .
\end{aligned}
$$

Here, $i$ denotes each constituent MML16 cloud, $M_{i}$ is the individual cloud mass, and $\left(l_{\mathrm{SG}}, b_{\mathrm{SG}}\right)$ are the massweighted mean Galactic coordinates of the host SFCGMC complex. The quantities $\left(l_{c, i}, b_{c, i}\right)$ are the Galactic coordinates of the 2 semi-major and 2 semi-minor vertices $(c=1-4)$ of each cloud, whose semi-major axis we define as $3 \sigma_{l}$ and semi-minor axis as $3 \sigma_{b}$.

Using the two eigenvalues $R_{\max }$ and $R_{\min }$ of $\phi$, we define the angular radius of each SFC-GMC complex as $R_{\text {ang }} \equiv\left(R_{\max } R_{\min }^{2}\right)^{1 / 3}$. We define the velocity dispersion of the SFC-GMC complex as

$$
\sigma_{\mathrm{v}, \mathrm{SG}}^{2}=\frac{\sum_{i} M_{i}\left(\delta v_{i}^{2}+\delta_{+} v_{i}^{2}+\delta_{-} v_{i}^{2}\right)}{\sum_{i} M_{i}}
$$

where $\delta v_{i}=v_{\text {cent }, \mathrm{i}}-v_{\mathrm{SG}}, \delta_{+} v_{i}=v_{\mathrm{cent}, \mathrm{i}}+\sigma_{v, i}-v_{\mathrm{SG}}$, and $\delta_{-} v_{i}=v_{\text {cent }, \mathrm{i}}-\sigma_{v, i}-v_{\mathrm{SG}}$ with $v_{\text {cent,i }}$ the central velocity of each cloud $i, v_{\mathrm{SG}}$ the mass-weighted velocity of the host SFC-GMC complex, and $\sigma_{v, i}$ the velocity dispersion of each cloud $i$. We have verified that the SFC-GMC complexes follow the $\sigma_{v}-M_{g}$ and $\alpha_{\mathrm{vir}}-M_{g}$ (where $\alpha_{\text {vir }} \equiv 5 \sigma_{v}^{2} R_{g} / G M_{g}$ is the cloud virial parameter in which $\left.R_{g}=d \tan \left(R_{\text {ang }}\right)\right)$ relations reported in MML16. The properties of SFC-GMC complexes are presented in Table 2. 
Table 1. MML16-SFC match (sorted by SFC luminosities)

\begin{tabular}{lcccccccccccccc}
\hline \hline $\begin{array}{c}\text { GMC } \\
\text { No. }\end{array}$ & $\begin{array}{c}l \\
(\mathrm{deg})\end{array}$ & $\begin{array}{c}\sigma_{l} \\
(\mathrm{deg})\end{array}$ & $\begin{array}{c}\mathrm{d} \\
(\mathrm{deg})\end{array}$ & $\begin{array}{c}\sigma_{b} \\
(\mathrm{deg})\end{array}$ & $\begin{array}{c}v \\
\left(\mathrm{~km} \mathrm{~s}^{-1}\right)\end{array}$ & $\begin{array}{c}\sigma_{v} \\
\left(\mathrm{~km} \mathrm{~s}^{-1}\right)\end{array}$ & $\begin{array}{c}d \\
(\mathrm{kpc})\end{array}$ & $\begin{array}{c}\text { SFC } \\
\text { No. }\end{array}$ & $\begin{array}{c}l \\
(\mathrm{deg})\end{array}$ & $\begin{array}{c}b \\
(\mathrm{deg})\end{array}$ & $\begin{array}{c}R \\
(\mathrm{deg})\end{array}$ & $\begin{array}{c}v \\
\left(\mathrm{~km} \mathrm{~s} \mathrm{~s}^{-1}\right)\end{array}$ & $\begin{array}{c}\sigma_{v} \\
\left.(\mathrm{~km} \mathrm{~s})^{-1}\right)\end{array}$ & $\begin{array}{c}d \\
(\mathrm{kpc})\end{array}$ \\
\hline 1726 & 336.57 & 0.14 & -0.24 & 0.14 & -88.23 & 5.64 & 10.25 & 227 & 336.41 & -0.02 & 0.50 & -79.00 & 20.33 & 10.63 \\
1761 & 336.17 & 0.14 & 0.05 & 0.18 & -68.67 & 7.31 & 11.07 & 227 & 336.41 & -0.02 & 0.50 & -79.00 & 20.33 & 10.63 \\
26 & 337.91 & 0.18 & 0.10 & 0.45 & -59.09 & 8.77 & 11.62 & 228 & 337.85 & -0.20 & 0.29 & -50.35 & 11.46 & 12.05 \\
279 & 337.48 & 0.19 & -0.07 & 0.39 & -54.86 & 6.90 & 11.81 & 228 & 337.85 & -0.20 & 0.29 & -50.35 & 11.46 & 12.05 \\
440 & 338.15 & 0.22 & -0.12 & 0.25 & -49.99 & 7.57 & 12.09 & 228 & 337.85 & -0.20 & 0.29 & -50.35 & 11.46 & 12.05 \\
171 & 29.94 & 0.19 & -0.24 & 0.20 & 101.81 & 4.41 & 8.37 & 68 & 30.04 & -0.24 & 0.33 & 99.40 & 5.82 & 8.55 \\
388 & 30.01 & 0.13 & -0.21 & 0.29 & 94.32 & 4.76 & 8.93 & 68 & 30.04 & -0.24 & 0.33 & 99.40 & 5.82 & 8.55 \\
583 & 30.26 & 0.23 & -0.44 & 0.15 & 102.03 & 5.21 & 6.44 & 68 & 30.04 & -0.24 & 0.33 & 99.40 & 5.82 & 8.55 \\
1054 & 305.62 & 0.32 & -0.24 & 0.27 & -42.13 & 4.43 & 4.48 & 182 & 305.66 & -0.07 & 0.61 & -39.10 & 9.38 & 6.24 \\
678 & 358.63 & 0.24 & -0.30 & 0.09 & -1.41 & 9.37 & 15.24 & 274 & 358.54 & -0.48 & 0.15 & -2.60 & 11.65 & 15.24 \\
\hline
\end{tabular}

NotE-Coordinates and velocities of SFCs and MML16 GMCs matched to each other. The left 8 columns are properties of MML16 clouds while the right 7 columns are properties of the matched SFCs. This table is published in its entirety in the electronic edition.

Table 2. SFC-GMC complexes (sorted by luminosities)

\begin{tabular}{lccccccccccc}
\hline \hline $\begin{array}{c}\text { SFC } \\
\text { No. }\end{array}$ & $\begin{array}{c}l \\
(\mathrm{deg})\end{array}$ & $\begin{array}{c}b \\
(\mathrm{deg})\end{array}$ & $\begin{array}{c}v \\
\left(\mathrm{~km} \mathrm{~s}^{-1}\right)\end{array}$ & $\begin{array}{c}\sigma_{v} \\
\left(\mathrm{~km} \mathrm{~s}^{-1}\right)\end{array}$ & $\begin{array}{c}R_{\mathrm{ang}} \\
(\mathrm{deg})\end{array}$ & $\begin{array}{c}R_{\mathrm{max}} \\
(\mathrm{deg})\end{array}$ & $\begin{array}{c}R_{\text {min }} \\
(\mathrm{deg})\end{array}$ & $\begin{array}{c}R_{\text {gal }} \\
(\mathrm{kpc})\end{array}$ & $\begin{array}{c}d \\
(\mathrm{kpc})\end{array}$ & $\begin{array}{c}W_{\mathrm{CO}} \\
\left(\mathrm{K} \mathrm{km} \mathrm{s}^{-1}\right)\end{array}$ & $\begin{array}{c}M_{g} \\
\left(M_{\odot}\right)\end{array}$ \\
\hline 227 & 336.30 & -0.05 & -75.34 & 10.80 & 0.35 & 0.41 & 0.33 & 4.56 & 10.79 & $3.94 \mathrm{e}+02$ & $9.47 \mathrm{e}+05$ \\
228 & 337.90 & -0.02 & -55.00 & 7.65 & 0.58 & 0.81 & 0.49 & 5.08 & 11.82 & $2.93 \mathrm{e}+03$ & $8.49 \mathrm{e}+06$ \\
68 & 30.01 & -0.25 & 98.41 & 5.34 & 0.42 & 0.52 & 0.38 & 4.44 & 8.42 & $2.08 \mathrm{e}+03$ & $2.97 \mathrm{e}+06$ \\
111 & 79.18 & 0.45 & -3.21 & 3.48 & 0.95 & 1.00 & 0.93 & 8.77 & 4.25 & $9.64 \mathrm{e}+03$ & $3.51 \mathrm{e}+06$ \\
274 & 358.63 & -0.30 & -1.41 & 9.37 & 0.24 & 0.47 & 0.17 & 6.75 & 15.24 & $4.83 \mathrm{e}+02$ & $2.33 \mathrm{e}+06$ \\
2 & 0.14 & -0.64 & 15.44 & 3.30 & 0.29 & 0.44 & 0.24 & 0.25 & 8.25 & $5.44 \mathrm{e}+02$ & $7.69 \mathrm{e}+05$ \\
249 & 347.78 & 0.08 & -95.96 & 6.88 & 0.33 & 0.42 & 0.30 & 2.70 & 10.32 & $4.23 \mathrm{e}+02$ & $9.33 \mathrm{e}+05$ \\
110 & 76.56 & 0.25 & -1.04 & 3.75 & 0.91 & 1.07 & 0.84 & 8.68 & 4.59 & $7.99 \mathrm{e}+03$ & $3.43 \mathrm{e}+06$ \\
72 & 31.02 & -0.09 & 102.25 & 5.59 & 0.45 & 0.57 & 0.40 & 4.42 & 6.69 & $1.34 \mathrm{e}+03$ & $1.24 \mathrm{e}+06$ \\
191 & 311.67 & 0.10 & -50.97 & 5.53 & 0.47 & 0.56 & 0.43 & 6.52 & 7.08 & $2.60 \mathrm{e}+03$ & $2.69 \mathrm{e}+06$ \\
\hline
\end{tabular}

Note-The order of SFC Nos. appear shuffled compared to Table 1 because we adopt mass-weighted distances of matched GMCs for each SFC here. This table is published in its entirety in the electronic edition.

\section{CALCULATING STELLAR MASS FROM FREE-FREE FLUX}

We aim to measure the spread in the distribution of clouds' star formation efficiencies $\epsilon$ (equation 1) and star formation rates $\epsilon_{\mathrm{ff}}$ (equation 2). We will compare the observed scatter with what is expected from turbulence-regulated star formation (Krumholz \& McKee 2005, KM05 from hereon).

The mass of stars associated with each cloud is evaluated from the WMAP free-free fluxes. We provide a brief summary of photometry here; readers interested in more details are referred to Lee et al. (2012). For a given $W M A P$ free-free source (the large $\sim 1-2^{\circ}$ wide regions identified by their peak free-free flux in WMAP; see Murray \& Rahman 2010 for more detail), we first perform aperture photometry to compute the total freefree flux. The total flux is divided into constituent SFCs (and by extension constituent SFC-GMC complexes), proportional to the relative SFC $8 \mu \mathrm{m}$ fluxes computed from Spitzer GLIMPSE and MSX images.

The gas mass of the cloud is calculated from the $\mathrm{CO}$ flux $W_{\mathrm{CO}}$. A measure of $\epsilon$ can then be probed by the 
flux ratio free-free $f_{\nu}^{\text {br }}$ over $W_{\mathrm{CO}}$ :

$$
\epsilon_{\mathrm{br}} \equiv \frac{a f_{\nu}^{\mathrm{br}} / W_{\mathrm{CO}}}{1+a f_{\nu}^{\mathrm{br}} / W_{\mathrm{CO}}}
$$

where the subscript br stands for Bremsstrahlung.

To understand the constant $a$, we review how $f_{\nu}$ 's are converted to the mass in live stars $M_{\star}$ and how $f_{\mathrm{CO}}$ 's are converted to the gas mass $M_{g}$.

A cloud with $f_{\nu}^{\text {br }}$ located at a distance $D$ has a luminosity $L_{\nu}^{\mathrm{br}}=4 \pi D^{2} f_{\nu}^{\mathrm{br}}$. Powering this luminosity requires a streaming rate of ionizing photons of $\mathcal{Q}=$ $1.34 \times 10^{26}\left(L_{\nu} / \mathrm{erg} \mathrm{s}^{-1} \mathrm{~Hz}^{-1}\right) \mathrm{s}^{-1}$. The ionizing luminosity is converted to $M_{\star}$ using the ratio of $\mathcal{Q}$ to $M_{\star}$ averaged over the modified Muench initial mass function (IMF) from Murray \& Rahman (2010):

$$
\frac{\left\langle m_{*}\right\rangle}{\langle q\rangle}=1.6 \times 10^{-47} \mathrm{~s}^{-1} \mathrm{M}_{\odot} .
$$

The live stellar mass in the cloud is then $M_{\star}=$ $1.37 \mathcal{Q}\left(\left\langle m_{\star}\right\rangle /\langle q\rangle\right)$ where the numerical factor 1.37 accounts for the absorption of ionizing photons by dust (which compete with hydrogen atoms as a sink of ionizing photons) following McKee \& Williams (1997).

The conversion between $W_{\mathrm{CO}}$ and the gas mass $M_{g}$ is given by equation (3). We can now define the constants $a$ and $b$ :

$$
\begin{aligned}
a & =\frac{M_{\star} / f_{\nu}^{\mathrm{br}}}{M_{g} / W_{\mathrm{CO}}} \\
& =\frac{4 \pi \times 10^{-23} \times\left(\mathcal{Q} / L_{\nu}\right) \times 1.37 \times\left(\left\langle m_{\star}\right\rangle /\langle q\rangle\right)}{X_{\mathrm{CO}} 2 \mu m_{\mathrm{H}} \tan (\delta)^{2}},
\end{aligned}
$$

where $10^{-23}$ is the conversion factor from jansky to cgs units, $X_{\mathrm{CO}}=2 \times 10^{20} \mathrm{~cm}^{-2} \mathrm{~K}^{-1} \mathrm{~s}, \mu=1.36$ to take into account helium, and $\delta=0.125^{\circ}$ is the pixel scale. Note the distance $D$ does not appear in the expression for $\epsilon$. Any error in the distance measurement will therefore not affect the scatter in the star formation efficiency.

Assuming all star clusters to follow a universal IMF, our computed stellar mass is reliable for massive clusters $\left(M_{\star} \geq 10^{4} M_{\odot} ;\right.$ Krumholz et al. 2015) that sample their IMFs well. There may also be variations in the IMF across different clusters (see, e.g., Dib 2014). Both the poor sampling of and the variations in the IMF introduce a scatter in the inferred stellar mass and by extension star formation efficiencies. We quantify the scatter by computing $\sigma$ in the ratio between the stellar mass reported by surveys of young stellar objects (YSOs) and the stellar mass we compute from the free-free fluxes for known clusters (see Figure 2). We find $\sigma=0.22$ dex.

\section{STAR FORMATION EFFICIENCY}

We use the measured scatter $\sigma_{\log \epsilon}$ as one metric to test various models of star formation rate. Using the efficiency of star formation appears to be particularly

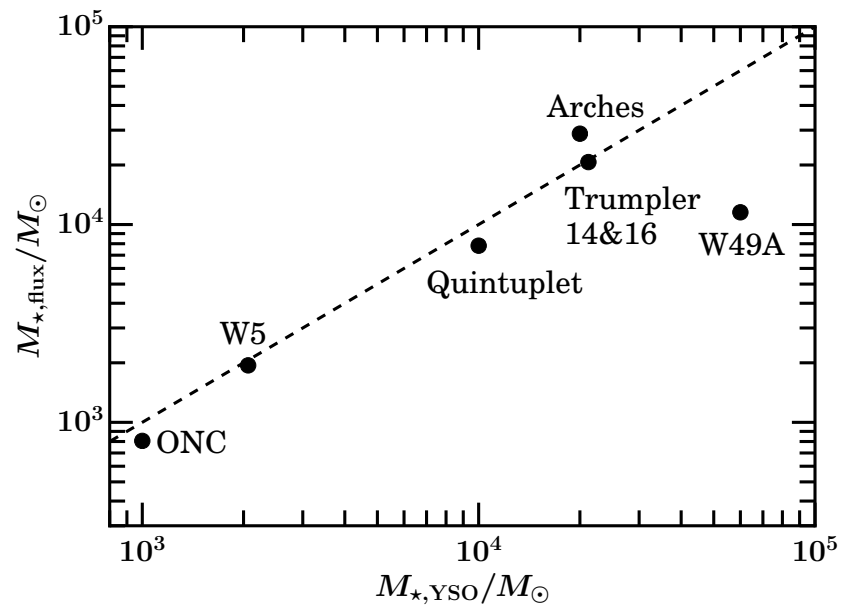

Figure 2. Comparison between the stellar mass of known young clusters as probed by counting young stellar objects (YSOs) and the stellar mass in the corresponding SFCs as probed by free-free flux. The dashed line delineates $M_{\star, \text { flux }}=M_{\star, \mathrm{YSO}}$. We adopt YSO counts for the Orion nebula cluster (ONC) from Da Rio et al. (2012), W5 from Koenig et al. (2008), Quintuplet and Arches from Portegies Zwart et al. (2010) and references therein, Trumpler 14 \& 16 from Portegies Zwart et al. (2010) and Wolk et al. (2011), respectively, and W49A from Homeier \& Alves (2005).

advantageous since the measured value of $\epsilon$ is independent of distance, so that errors in the distance determination to either GMCs or star clusters do not introduce any scatter in the distribution of $\epsilon$. However, the comparison of $\sigma_{\log \epsilon}$ to the prediction of the constant star formation rate per free-fall time theory do require the use of a distance estimate, somewhat reducing the attractiveness of this particular metric for our purposes.

Figure 3 shows the star formation efficiency $\epsilon$ as a function of the mass of the host GMCs (left panels) and the histogram of $\epsilon$ (right panels). We recover the result of Mooney \& Solomon (1988) that the ratio of FIR to $\mathrm{CO}$ flux has a very broad distribution. Specifically, we find that $\epsilon$ ranges over three orders of magnitude. After correcting for the correlation between $\epsilon$ and the total mass, we calculate the scatter $\sigma_{\log \epsilon}$ as defined in Section 3: about $68.3 \%$ of the data fall within 0.79 dex.

There are a number of sources of scatter in $\epsilon$ that stem from measurement uncertainties. Ionizing photons can travel surprising long distance so if the aperture chosen to measure the free-free flux is too small, $\epsilon_{\mathrm{br}}$ will be underestimated. If there are nearby $(\sim 100 \mathrm{pc})$ sources of ionizing radiation and the aperture is overly large, $\epsilon_{\mathrm{br}}$ will be overestimated. Miss-identificationsregions which are not physically associated are nevertheless cross-correlated - will also introduce artificial scatter into the value of $\epsilon$.

The aperture for free-free measurements are limited by the low resolution of the WMAP free-free map. We 

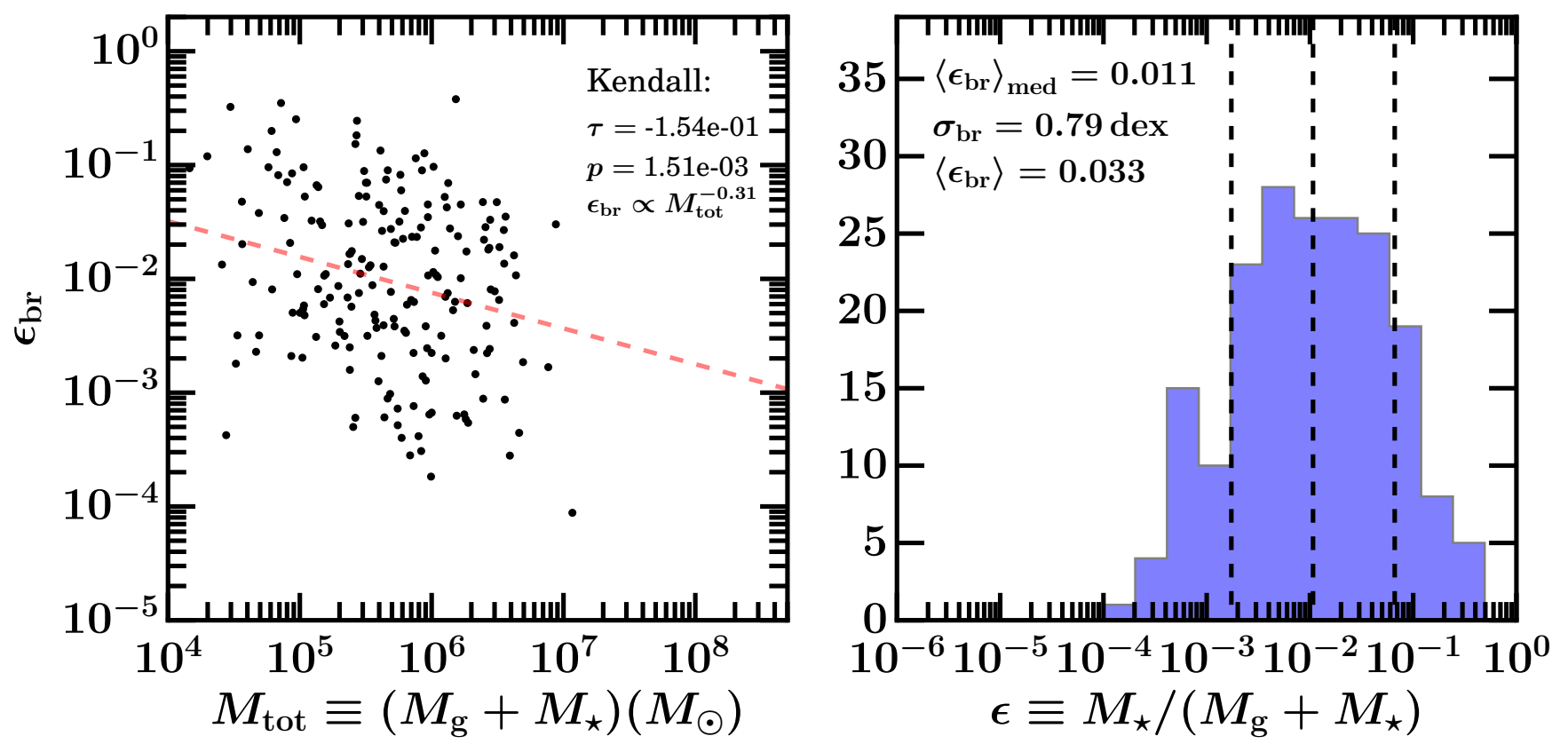

Figure 3. Left: the star formation efficiency $\epsilon_{\mathrm{br}}$ (the subscript br stands for Bremsstrahlung) of 191 SFC-GMC complexes plotted as a function of total (gas plus stellar) mass. The correlation between $\epsilon$ and $M_{\text {tot }}$ is statistically significant; we draw with the red dashed line the least-square fit correlation. Right: histograms of $\epsilon$ calculated with free-free flux. The middle dashed line represents the median $\epsilon$ while the dashed lines to the left and right illustrate $\sigma$. The mean $\epsilon \sim 0.03$ is in rough agreement with that of nearby clouds (e.g., Evans et al. 2009; Gutermuth et al. 2011). The full width of the distribution spans $\sim 3$ orders of magnitude. Since the p-values of Kendall's $\tau$ test is smaller than 0.05, we remove the annotated least-square fit between $\epsilon$ and $M_{\text {tot }}$ before estimating the $\sigma$.

have chosen to assign free-free fluxes of SFCs according to their relative $8 \mu \mathrm{m}$ fluxes. Varying the aperture size of SFCs, Lee et al. (2012) quote an $88 \%$ measurement error in $8 \mu \mathrm{m}$, which we adopt for free-free fluxes of all GMCs. Propagating these errors in flux measurement, each cloud has an error of $\delta \log \epsilon=0.38$ dex. For the entire sample of 191 star-forming clouds, measurement errors contribute $\Delta \sigma_{\log \epsilon_{\delta \text { flux }}}=0.03$ dex.

The conversion factor between free-free luminosities and stellar masses depends on the IMF. In Section 3, we estimated the errors introduced by the poor sampling of the IMF and the physical variations in the IMF as 0.22 dex. Adding the two sources of error in quadrature, we find

$$
\Delta \sigma_{\log \epsilon}=0.22 \text { dex. }
$$

Combining all our error estimates, we arrive at our final error estimate for the width of the distribution of star formation efficiency:

$$
\sigma_{\log \epsilon_{\mathrm{br}}}=0.79 \pm 0.22 \mathrm{dex}
$$

This is the first of two main observational results in this paper: the distribution of star formation efficiencies $\log \epsilon$ is very broad.

\section{STAR FORMATION RATE PER FREE FALL TIME $\epsilon_{\mathrm{FF}}$}

The quantity $\epsilon_{\mathrm{ff}}$ is an estimate of the star formation rate, normalized to the free-fall time of the star-forming region (GMCs in our case, see equation 2). We will measure the scatter in the distribution of $\epsilon_{\mathrm{ff}}$ and compare it against what is expected from various models of star formation (the main test case being turbulence-regulated star formation proposed by Krumholz \& McKee 2005, KM05 and Hennebelle \& Chabrier 2011, HC11).

Using the definition of $\epsilon_{\mathrm{br}}$ from equations 9, equation 2 can be rewritten as

$$
\epsilon_{\mathrm{ff}}^{\mathrm{br}}=\epsilon_{\mathrm{br}} \frac{\tau_{\mathrm{ff}}}{\left\langle t_{\mathrm{ms}, \mathrm{q}}\right\rangle},
$$

where $\left\langle t_{\mathrm{ms}, \mathrm{q}}\right\rangle \approx 3.9 \mathrm{Myrs}$ is the Q-weighted mainsequence lifetime of $\mathrm{O}$ stars.

We calculate the free-fall time $\tau_{\mathrm{ff}}$ using the ellipsoidal volume found by assuming that the length along the projected radial direction to be equal to the shorter of the two axes in the plane of the sky.

Unlike the measurement of $\epsilon$, the measurement of $\epsilon_{\mathrm{ff}}$ depends on the distance to the source, via the free-fall time $\tau_{\mathrm{ff}}$ factor in equation (14). Thus we expect that the scatter in $\epsilon$ is smaller than that of $\epsilon_{\mathrm{ff}}$, and this is what we find. The scatter in the GMC free-fall time is $\sigma_{\log \tau_{\mathrm{ff}}} \approx 0.27 \mathrm{dex}$. This is much smaller than the scatter in $\epsilon_{\mathrm{br}}, \sigma_{\log \epsilon_{\mathrm{br}}}=0.79$ dex.

Part of the scatter in $\tau_{\mathrm{ff}}$ is due to uncertainties in our distance measurements, but most of it is intrinsic. More 
precisely, $\tau_{\mathrm{ff}} \propto f_{\mathrm{CO}}^{-1 / 2} D^{1 / 2}$. The errors in the measurement of distances are typically $\sim 35 \%$ (Lee et al. 2012). Propagating errors in both the flux and distance measurements, we find errors of $\delta \log \tau_{\mathrm{ff}}=0.22$ dex for each cloud. Combining this with the flux measurement errors in $\epsilon, \delta \log \epsilon_{\mathrm{ff}}=0.44 \mathrm{dex}$. For the entire sample of 191 SFC-GMCs, the measurement errors are $\Delta \sigma_{\log \epsilon_{\mathrm{ff}}}=0.03$ dex. Combining the measurement error with the error introduced by the poor sampling and the variations in the IMF $\sigma=0.22$ dex,

$$
\sigma_{\log \epsilon_{\mathrm{ff}}^{\mathrm{br}}}=0.91 \pm 0.22 \mathrm{dex}
$$

This is our second major observational result: the distribution of star formation rate per free fall time $\epsilon_{\mathrm{ff}}$ is very broad.

We can compare this directly to the prediction of
KM05 and HC11, using the properties of the GMCs we used to measure $\epsilon_{\mathrm{ff}}$ (see Section 6). We find $\sigma_{\log \epsilon_{\text {ff }, \mathrm{KM} 05}}=0.24 \mathrm{dex}$ and $\sigma_{\log \epsilon_{\text {,f }, \mathrm{HC} 11}}=0.13$ or 0.12 dex, depending on the choice of parameters (to be elaborated in Section 6). The model-inferred scatter in $\epsilon_{\mathrm{ff}}$ are $3,3.5$, and 3.6 standard deviations from the observed value of $0.91 \pm 0.22$ dex (see Figure 4).

The fact that the measured scatter in $\epsilon_{\mathrm{ff}}$ is significantly larger than the prediction of either KM05 or HC11 model, which account for variations in the Mach number and virial parameter of the host GMC (but ignores any explicit time dependence in the rate of star formation) is a strong evidence that the model is incomplete. We discuss in more detail in the next section.

We summarize the result of our calculations of $\epsilon$ and $\epsilon_{\mathrm{ff}}$ in Table 3.

Table 3. Star formation properties of SFC-GMC complexes

\begin{tabular}{|c|c|c|c|c|c|c|c|c|c|c|}
\hline $\begin{array}{l}\text { SFC } \\
\text { No. }\end{array}$ & $\begin{array}{c}\sigma_{v} \\
\left(\mathrm{~km} \mathrm{~s}^{-1}\right)\end{array}$ & $\begin{array}{c}d \\
(\mathrm{kpc})\end{array}$ & $\begin{array}{c}R \\
(\mathrm{pc})\end{array}$ & $\begin{array}{c}Q \\
\left(\mathrm{~s}^{-1}\right)\end{array}$ & $\begin{array}{c}M_{g} \\
\left(M_{\odot}\right)\end{array}$ & $\begin{array}{c}\Sigma_{g} \\
\left(M_{\odot} \mathrm{pc}^{-2}\right)\end{array}$ & $\epsilon_{\mathrm{br}}$ & $\epsilon_{\mathrm{ff}, \mathrm{br}}$ & $\begin{array}{c}\tau_{\mathrm{ff}} \\
(\mathrm{Myr})\end{array}$ & $\alpha_{\text {vir }}$ \\
\hline 227 & 10.80 & 10.79 & 66.84 & $3.64 \mathrm{e}+52$ & $9.47 \mathrm{e}+05$ & $6.31 \mathrm{e}+01$ & $3.79 \mathrm{e}-01$ & $9.14 \mathrm{e}-01$ & 9.32 & $9.58 \mathrm{e}+00$ \\
\hline 228 & 7.65 & 11.82 & 119.13 & $1.65 \mathrm{e}+52$ & $8.49 \mathrm{e}+06$ & $1.61 \mathrm{e}+02$ & $3.01 \mathrm{e}-02$ & $5.77 \mathrm{e}-02$ & 7.41 & $9.58 \mathrm{e}-01$ \\
\hline 68 & 5.34 & 8.42 & 61.64 & $9.50 \mathrm{e}+51$ & $2.97 \mathrm{e}+06$ & $2.23 \mathrm{e}+02$ & $4.72 \mathrm{e}-02$ & $5.69 \mathrm{e}-02$ & 4.66 & $6.90 \mathrm{e}-01$ \\
\hline 111 & 3.48 & 4.25 & 70.60 & $8.28 \mathrm{e}+51$ & $3.51 \mathrm{e}+06$ & $2.18 \mathrm{e}+02$ & $3.52 \mathrm{e}-02$ & $4.79 \mathrm{e}-02$ & 5.26 & $2.83 \mathrm{e}-01$ \\
\hline 274 & 9.37 & 15.24 & 64.29 & $7.24 \mathrm{e}+51$ & $2.33 \mathrm{e}+06$ & $1.51 \mathrm{e}+02$ & $4.73 \mathrm{e}-02$ & $6.86 \mathrm{e}-02$ & 5.61 & $2.82 \mathrm{e}+00$ \\
\hline 2 & 3.30 & 8.25 & 42.39 & $7.00 \mathrm{e}+51$ & $7.69 \mathrm{e}+05$ & $1.13 \mathrm{e}+02$ & $1.27 \mathrm{e}-01$ & $1.71 \mathrm{e}-01$ & 5.22 & $6.98 \mathrm{e}-01$ \\
\hline 249 & 6.88 & 10.32 & 59.93 & $6.25 \mathrm{e}+51$ & $9.33 \mathrm{e}+05$ & $7.36 \mathrm{e}+01$ & $9.66 \mathrm{e}-02$ & $1.99 \mathrm{e}-01$ & 7.97 & $3.54 \mathrm{e}+00$ \\
\hline 110 & 3.75 & 4.59 & 73.23 & $6.04 \mathrm{e}+51$ & $3.43 \mathrm{e}+06$ & $1.88 \mathrm{e}+02$ & $2.68 \mathrm{e}-02$ & $3.90 \mathrm{e}-02$ & 5.62 & $3.49 \mathrm{e}-01$ \\
\hline 72 & 5.59 & 6.69 & 52.90 & $5.81 \mathrm{e}+51$ & $1.24 \mathrm{e}+06$ & $8.71 \mathrm{e}+01$ & $6.95 \mathrm{e}-02$ & $1.03 \mathrm{e}-01$ & 5.73 & $1.55 \mathrm{e}+00$ \\
\hline 191 & 5.53 & 7.08 & 57.79 & $5.79 \mathrm{e}+51$ & $2.69 \mathrm{e}+06$ & $2.34 \mathrm{e}+02$ & $3.30 \mathrm{e}-02$ & $3.80 \mathrm{e}-02$ & 4.45 & $7.67 \mathrm{e}-01$ \\
\hline
\end{tabular}

Note- The physical radius $R$ is defined as $d \tan \left(R_{\text {ang }}\right)$. The gas surface density $\Sigma_{g} \equiv M_{g} /\left(d \tan \left(\pi R_{\max } R_{\min }\right)\right)^{2}$ while the virial parameter $\alpha_{\text {vir }} \equiv 5 \sigma_{v}^{2} R / G M_{g}$ where $G$ is the gravitational constant. This table is published in its entirety in the electronic edition.

\section{IMPLICATIONS OF THE LARGE SCATTER IN THE EFFICIENCY AND THE RATE OF STAR FORMATION}

We discuss a number of ideas regarding the physical processes that regulate the rate of star formation in light of our observational results.

\subsection{Turbulence regulated star formation}

KM05 present a semi-analytic model of $\epsilon_{\mathrm{ff}}$ based on the idea of turbulence-regulated star formation. They posit that within a turbulent cloud whose density distribution is well-characterized by a log-normal distribution - with its width governed by the Mach number - only the regions of densities larger than some critical value will collapse to form stars. This crit- ical value depends on both the virial parameter and the Mach number. Clouds with larger $\alpha_{\text {vir }}$ and $\mathcal{M}$ are harder to collapse so the critical $\rho$ for such clouds will be larger, leading to a smaller star formation rate per free-fall time $\epsilon_{\mathrm{ff}}$.

Assuming that the log-normal density distribution established by turbulence is maintained over the cloud lifetime (but see e.g., Vázquez-Semadeni et al. 2008, Cho \& Kim 2011, Kritsuk et al. 2011, Collins et al. 2012, Federrath \& Klessen 2013 for the evidence of dynamic density distributions in star-forming regions), KM05 find

$$
\epsilon_{\mathrm{ff}, 0}=0.014\left(\frac{\alpha_{\mathrm{vir}}}{1.3}\right)^{-0.68}\left(\frac{\mathcal{M}}{100}\right)^{-0.32}
$$

(their equation 30). We append a subscript 0 to $\epsilon_{\mathrm{ff}}$ 

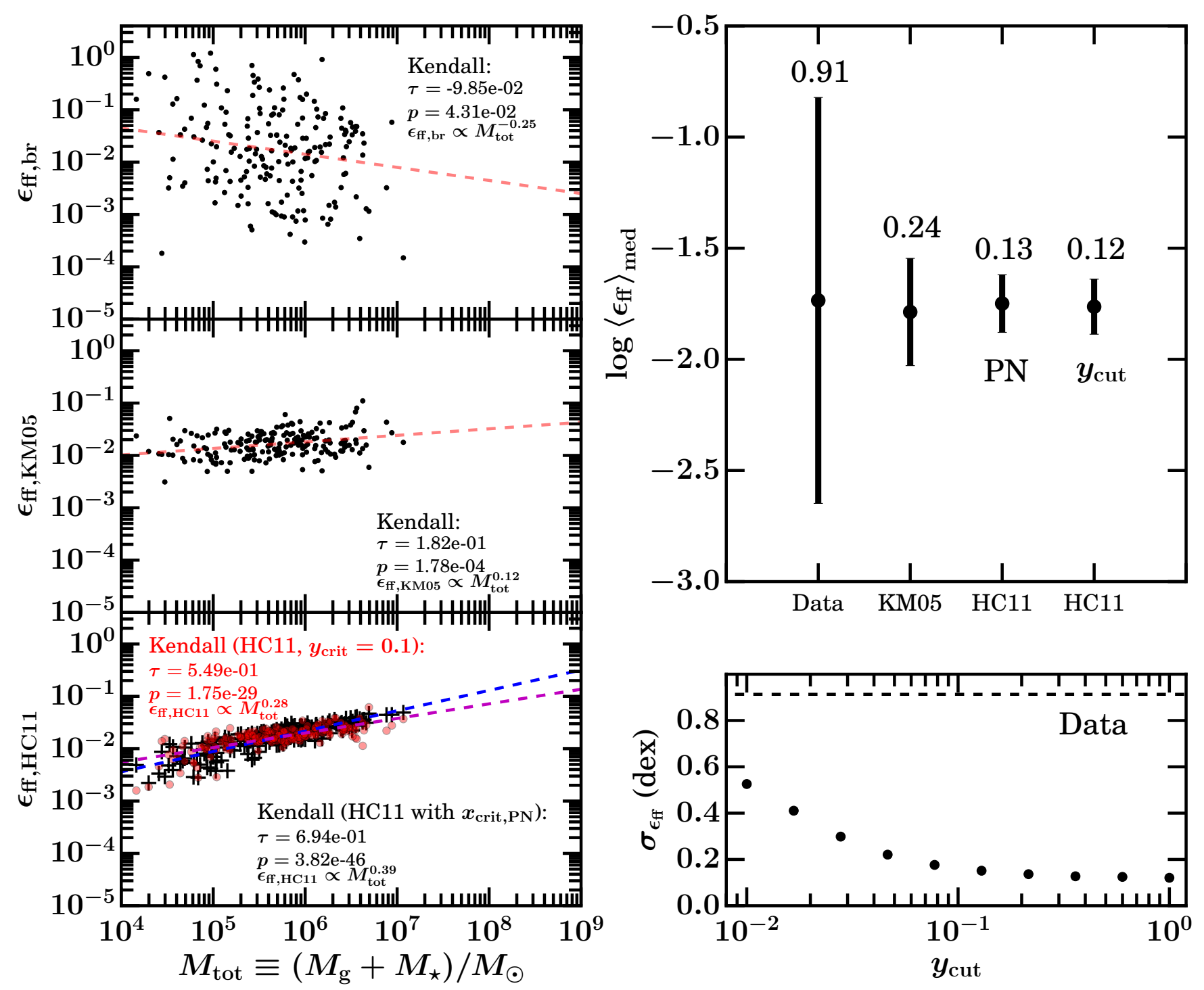

Figure 4. Top left: the star formation rate per free-fall time $\epsilon_{\mathrm{ff}}$ of 191 SFC-GMC complexes as a function of the total (gas + star) mass. We see a correlation between $\epsilon_{\mathrm{ff}}$ and the total mass that is marginally statistically significant; the least-square fit correlation is drawn with the red dashed line. Middle left: $\epsilon_{\mathrm{ff}}$ predicted by equation 30 of Krumholz \& McKee (2005) (equation 16 in this paper). We see a statistically significant correlation between $\epsilon_{\mathrm{ff}, \mathrm{KM} 05}$ and the total mass (also drawn with the red dashed line). Bottom left: $\epsilon_{\mathrm{ff}}$ predicted by the multi-freefall theory of star formation from Hennebelle \& Chabrier (2011, labeled as HC11), their equation 8 (our equation 20; see also Federrath \& Klessen 2012). Following HC11, we compute the model-inferred $\epsilon_{\mathrm{ff}, \mathrm{HC} 11}$ using two different criteria on the critical gas density over which star formation occurs: the local Jeans length is equal to the thickness of a shocked layer for a given Mach number (Padoan \& Nordlund 2011, PN); and the local Jeans length is equal to some prescribed fraction $y_{\text {cut }}$ of the cloud size (Hennebelle \& Chabrier 2011, $y_{\text {cut }}$ ); we adopt their recommended $y_{\text {cut }}=0.1$. We see a strong positive correlation between $\epsilon_{\mathrm{ff}, \mathrm{HC} 11}$ and $M_{\text {tot }}$ in contrast to marginal negative correlation observed in the data (upper left panel). The least-square fit correlations are drawn in blue for PN and in magenta for $y_{\text {cut }}$. We normalized $\epsilon_{\mathrm{ff}, \mathrm{HC11}}$ such that its median matches that of the observed. We plot the median $\epsilon_{\mathrm{ff}}$ in the upper right panel with the errorbar indicating $\sigma$. The observed scatter in $\epsilon_{\mathrm{ff}}=0.91$ dex is significantly larger than what any static model predicts: 0.24 dex (KM05), 0.13 dex $(\mathrm{HC} 11, \mathrm{PN})$, and 0.12 dex (HC11, $\left.y_{\text {cut }}\right)$. All $\sigma$ 's are measured after correcting $\epsilon_{\mathrm{ff}}$ for its correlation with the total mass (annotated in the left panels). Bottom right panel shows that no $y_{\text {cut }}$ within a reasonable range can reconcile HC11 model with the enormous scatter in $\epsilon_{\mathrm{ff}}$ observed in the data (shown as the dashed line). 


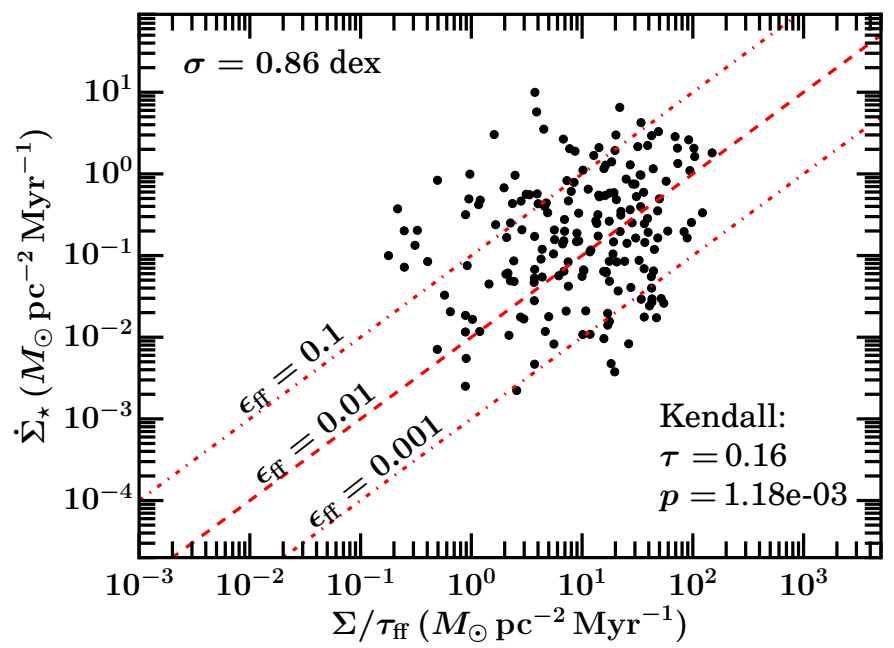

Figure 5. Star formation rate per unit area vs. the cloud gas surface density divided by the free-fall time, analogous to Figure 3 of Krumholz et al. (2012). The red dashed line delineates the volumetric star formation law proposed by Krumholz et al. (2012) assuming a constant $\epsilon_{\mathrm{ff}}=0.01$. We draw two different constant $\epsilon_{\mathrm{ff}}(0.1$ and 0.001$)$ in red dotdashed lines for reference. We find a significant correlation between $\Sigma_{\star}$ and $\Sigma / \tau_{\text {ff }}$. However, the scatter about the volumetric star formation law $\dot{\Sigma}_{\star} \propto \Sigma / \tau_{\mathrm{ff}}$ is large: 0.86 dex.

to emphasize that their model assumes that the star formation rate is time-independent. We see in Figure 4 that the KM05 model expects a scatter in $\epsilon_{\mathrm{ff}}, \sigma=0.24$ dex, significantly smaller than the observed $\sigma=0.91$ dex.

The KM05 model has been criticized for characterizing an entire cloud and its collapsing substructure by a single free-fall timescale. Star-forming substructures have varying densities and therefore different free-fall times (see e.g., Hennebelle \& Chabrier 2011, Federrath \& Klessen 2012, and references therein). Hennebelle \& Chabrier (2011) present an analytic model of $\epsilon_{\mathrm{ff}}$, which takes into account not only the different free-fall time for each collapsing structure but also the recycling of turbulent flow over a dynamical time (see also Federrath \& Klessen 2012; Hennebelle \& Chabrier 2013):

$$
\epsilon_{\mathrm{ff}} \propto e^{3 \sigma_{\rho}^{2} / 8}\left[1+\operatorname{erf}\left(\frac{\sigma_{\rho}^{2}-\ln x_{\mathrm{crit}}}{2^{1 / 2} \sigma_{\rho}}\right)\right]
$$

where $\sigma_{\rho}$ is the spread in the density distribution, set by the cloud Mach number:

$$
\sigma_{\rho}^{2}=\ln \left(1+b^{2} \mathcal{M}^{2}\right)
$$

$(b=0.25$ for purely solenoidal driving and $b=1.0$ for purely compressive driving; we take $b=0.5$ ) and $x_{\text {crit }} \equiv \rho_{\text {crit }} / \rho_{0}$ is the critical density over which substructure begins to collapse normalized by the average bulk density of the cloud $\rho_{0}$. We experiment with two criteria for collapsing: if the local Jeans length becomes comparable to the local shock width (Padoan \& Nord- lund 2011, PN criterion):

$$
x_{\text {crit }} \simeq 0.067 \theta^{-2} \alpha_{\text {vir }} \mathcal{M}^{2}
$$

with $\theta=0.35$ and if the local Jeans length becomes comparable to some prescribed fraction $y_{\text {cut }}$ of the cloud size (Hennebelle \& Chabrier 2011, $y_{\text {cut }}$ criterion):

$$
x_{\text {crit }}=\frac{1}{5 y_{\text {cut }}} \frac{\alpha_{\text {vir }}}{\mathcal{M}^{2}}\left(1+\mathcal{M} y_{\text {cut }}^{p}\right) .
$$

Here, $p$ is the power-law scale from the size-linewidth relationship $\sigma \propto R^{p}$; we adopt $\mathrm{p}=0.5$. The normalization of equation 17 represents the efficiency at which a clump gas converts to a star, and we adjust it so that the median model-inferred $\epsilon_{\mathrm{ff}}$ matches that of the observed. The match warrants an unusually small normalization: 0.009 for the PN criterion and 0.002 for the $y_{\text {cut }}$ criterion, compared to the usual $0.02-0.05$.

These multi-freefall models of star formation show stronger dependence of $\epsilon_{\mathrm{ff}}$ on the Mach number compared to the KM05 model. It has been argued that the observed scatter in $\epsilon_{\mathrm{ff}}$ of Milky Way clouds can be explained if the clouds' Mach number or the scale of turbulence driving vary by $\gtrsim 2$ orders of magnitude (e.g., Federrath 2013; Chabrier et al. 2014). Figure 4 demonstrates that the velocity dispersion or the size of the clouds simply do not vary enough to reconcile even the multi-freefall model with the observed scatter in $\epsilon_{\mathrm{ff}}$ of the MML16 clouds. No reasonable choice of $y_{\text {cut }}$ can reproduce the large $\sigma=0.91$ dex. Furthermore, HC11 models predict a strong positive correlation between $\epsilon_{\mathrm{ff}}$ and the total mass in contrast to a weak negative correlation seen in the data.

Three facts emerge from our analysis that hints at the controlling parameters of star formation other than $\alpha_{\text {vir }}, \mathcal{M}$, and the scale of turbulence driving. First, the measured scatter is significantly larger than any theoretically expected scatter. Second, there is little systematic offset between the observed and the KM05 distribution of $\epsilon_{\mathrm{ff}}$. Third, an unusually small gas-to-core efficiency is required to match the median $\epsilon_{\mathrm{ff}}$ expected from the multi-freefall models to that observed. All three facts suggest the distribution of Milky Way cloud $\mathcal{M}$ and size is far too narrow to explain the observed scatter in $\epsilon_{\mathrm{ff}}$ with what the multi-freefall models predict.

Both the larger scatter and the lack of systematic offset between the observed and theoretical distribution of $\epsilon_{\mathrm{ff}}$ is also evident in Figure 5. The figure shows the star formation rate per unit area $\dot{\Sigma}_{\star}$ plotted against the ratio between the gas surface density and the free-fall time, $\Sigma / \tau_{\mathrm{ff}}$, a comparison advocated by Krumholz et al. (2012).

Like Krumholz et al. (2012), we find a statistically significant correlation between $\dot{\Sigma}_{\star}$ and $\Sigma / \tau_{\mathrm{fff}}$. A leastsquare fit produces $\dot{\Sigma}_{\star} \propto\left(\Sigma / \tau_{\mathrm{ff}}\right)^{0.3}$, compared to the 


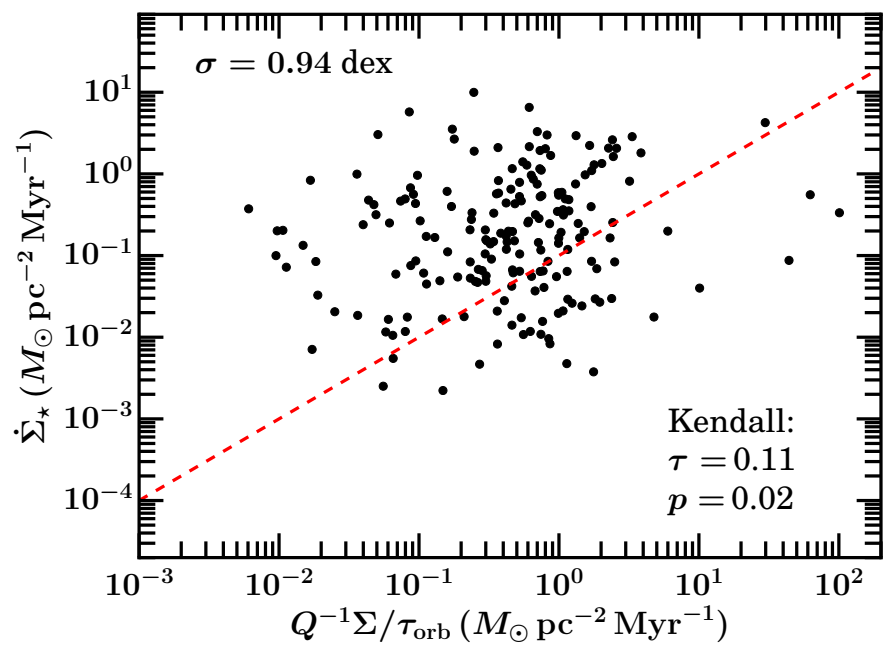

Figure 6. Star formation rate per unit area $\dot{\Sigma}_{\star}$ vs. the cloud surface density divided by the collision time $\sim \tau_{\text {orb }} Q$. The orbital time $\tau_{\text {orb }}=2 \pi R_{\text {gal }} / 220 \mathrm{~km} \mathrm{~s}^{-1}$ where $R_{\text {gal }}$ is the galactocentric radius and we assume a flat rotation curve $v(r)=220 \mathrm{~km} \mathrm{~s}^{-1}$. The red dashed line illustrates equation 21 with $\epsilon_{\mathrm{col}}=0.1$, assuming a flat rotation curve $(\beta=0)$. We see a strong correlation between $\dot{\Sigma}_{\star}$ and $Q^{-1} \Sigma / \tau_{\text {orb }}$ but the scatter about the model is still large: 0.92 dex.

linear relationship (the volumetric star formation law) reported by Krumholz et al. (2012).

We interpret the difference in the power-law index as the result of the large scatter in the observed value of $\epsilon_{\mathrm{ff}}$. As Figure 5 attests, the data points span more than an order of magnitude both above and below $\epsilon_{\mathrm{ff}}=0.01$, well beyond the expected uncertainty $\sim 3$ predicted by (Krumholz et al. 2012). We find a dispersion about the volumetric law of 0.86 dex. Similarly large scatters in star formation rates are observed in local molecular gas from Gould's belt (Evans et al. 2014) and in ATLASGAL clumps (Heyer et al. 2016). The sample of clouds assembled by Evans et al. (2014) falls squarely within the range $\epsilon_{\mathrm{ff}}=0.1$ and $\epsilon_{\mathrm{ff}}=0.001$. A few ATLASGAL clumps analyzed by Heyer et al. (2016) fall below $\epsilon_{\mathrm{ff}}=0.001$ but no clumps lie beyond $\epsilon_{\mathrm{ff}}=0.1$. Our GMC sample is more complete than the clump samples in either the Gould's belt or ATLASGAL clumps, which may explain part of the difference in the dispersions found in the different surveys.

\subsection{Collision-induced Star Formation}

An alternate form of star formation law envisions cloud-cloud collision to regulate the star formation rate on approximately orbital timescales (see e.g., Tan 2000; Suwannajak et al. 2014, and references therein):

$$
\dot{\Sigma}_{\star} \simeq \epsilon_{\mathrm{col}} Q^{-1}(1-0.7 \beta) \Sigma / \tau_{\text {orb }}
$$

where $\epsilon_{\text {orb }}=0.1$ is the rate at which gas is converted to stars for each collision, $Q \sim \sqrt{\alpha_{\text {vir }}}$ the Toomre parameter, $\tau_{\text {orb }}$ the orbital time, and $\beta$ is the logarithmic

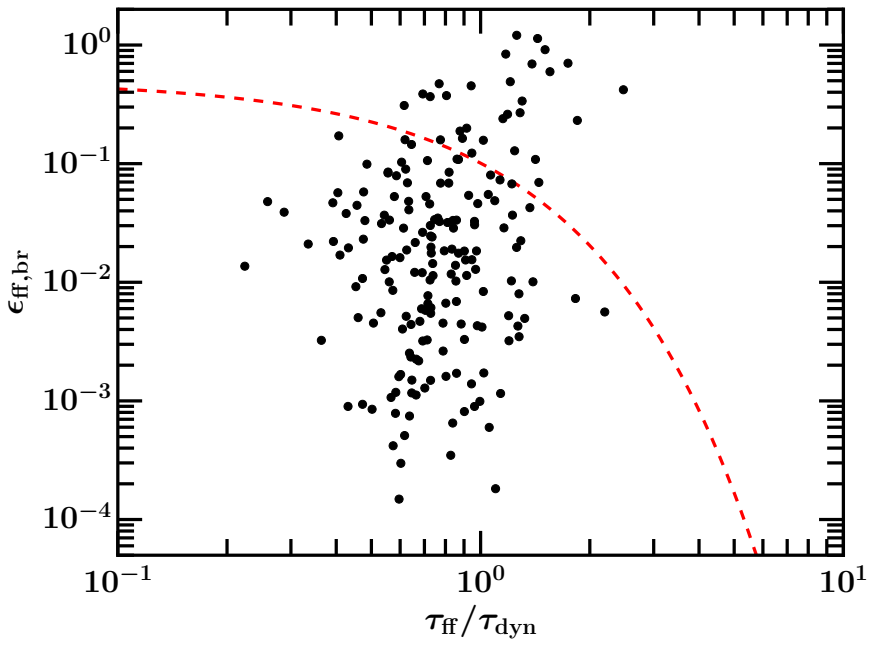

Figure 7. Star formation rate per free-fall time $\epsilon_{\mathrm{ff}}$ plotted against the ratio between the free-fall and the dynamical time, $\tau_{\mathrm{ff}} / \tau_{\text {dyn }}$. The star formation law in equation 22 is drawn as red dashed lines. The time ratio (equivalently, the square-root of the virial parameter) may be an important parameter related to the maximum possible $\epsilon_{\mathrm{ff}}$, but by itself it does not explain the large scatter in $\epsilon_{\mathrm{ff}}$.

derivative of the velocity profile. Assuming a flat rotation curve $(\beta=0)$, we show in Figure 6 that our star-forming clouds scatter about the collision-induced star formation law with a dispersion of $\sigma=0.94$ dex. As in Figure 5, the full min-mid or mid-max scatter is more than an order of magnitude; it is unlikely that the shear-velocity dependent term $(1-0.7 \beta)$ vary by more than a factor of order unity.

As with the volumetric star formation law, the large scatter about the collision-induced star formation law suggests there are extra parameters that control the rate at which gas is converted into stars.

\subsection{SFR Parametrized by Virial Parameter}

Padoan et al. (2012) studied magneto-hydrodynamic simulations of turbulent, star-forming gas, finding that $\epsilon_{\mathrm{ff}}$ depends exponentially on the ratio between the freefall and the dynamical time (which is equivalent to the square-root of the virial parameter):

$$
\epsilon_{\mathrm{ff}} \simeq \epsilon_{w} e^{\left(-1.6 \tau_{\mathrm{ff}} / \tau_{\mathrm{dyn}}\right)},
$$

where $\epsilon_{w}$ accounts for the mass loss due to proto-stellar winds and outflows, and $\tau_{\text {dyn }}$ is the dynamical time in their simulations.

Figure 7 shows the star formation rate per free-fall time for star-forming clouds, plotted as a function of $\tau_{\mathrm{ff}} / \tau_{\mathrm{dyn}}$ where $\tau_{\mathrm{dyn}}=R_{g} / \sigma_{\mathrm{GMC}}$ is the dynamical time of the host cloud. We do see a hint of a decrease in the upper envelope of the distribution of $\epsilon_{\mathrm{ff}}$ with increasing $\tau_{\text {ff }} / \tau_{\text {dyn }}$. However, many GMCs are more efficient at producing stars than what equation 22 would suggest. More strikingly, the $\epsilon_{\mathrm{ff}}$ values of GMCs span $\sim 3$ orders of 
magnitude below the prediction by Padoan et al. (2012). Padoan et al. (2012) noted that $\epsilon_{\mathrm{ff}}$ depended on the Alfvénic Mach number $\mathcal{M}_{a}$, with lower Alfvénic Mach number (stronger magnetic field for a given strength of turbulence) yielding smaller $\epsilon_{\mathrm{ff}}$, but only for $\mathcal{M}_{a} \gtrsim 5$; below this value, $\epsilon_{\mathrm{ff}}$ increases again (see their Figure 3). Their simple fitting formula (our equation 22) corresponds to the minimum star formation rates in their models with $\mathcal{M}_{a} \approx 5$. Can the variation in the magnetic field strength explain the large scatter in $\epsilon_{\mathrm{ff}}$ ?

Variations in $\mathcal{M}_{a}$ may explain those points that lie above the red dashed line (corresponding to equation 22 ) in Figure 7, but the extreme low values seen below the red dashed line cannot be explained by variations in the magnetic field strength. The Alfvénic Mach number can be estimated as $\mathcal{M}_{a} \sim \alpha_{\text {vir }}\left(M_{g} / M_{\Phi}\right)$ where $\alpha_{\text {vir }}$ is the virial parameter and $M_{\Phi}=0.12 \pi B\left(R_{g} / 2\right)^{2} / \sqrt{G}$ is the magnetic critical mass where $B$ is the magnetic field strength. SFC-GMC clouds typically have volumetric number densities of $n_{\mathrm{H}} \sim 10-200 \mathrm{~g} \mathrm{~cm}^{-3}$ so their $B \sim 1-$ $10 \mu \mathrm{G}$ (Crutcher 2012); we find that these clouds have $M_{g} / M_{\phi} \sim 10-100$. The median $\alpha_{\text {vir }} \sim 0.76$ with a scatter of 0.32 dex. We estimate $\mathcal{M}_{a} \sim 4-200$.

The simulations of Padoan et al. (2012) show that an order of magnitude change in $\mathcal{M}_{a}$ results in a factor of $\sim 3$ change in $\epsilon_{\mathrm{ff}}$. Taking the variations in $\mathcal{M}_{a}$ of the star-forming clouds into account, we expect an upward scatter from the reference value given by equation 22 by factors of $\sim 10$.

\subsection{Constant vs. Time-dependent star formation rate per free fall time}

All four models described in Section 6-singlefreefall turbulence-regulated, multi-freefall turbulenceregulated, collision-induced, and parametrization by virial parameter - assume a star formation rate that has no explicit dependence on time. These models predict that any observed scatter in $\epsilon_{\mathrm{ff}}$ should arise from variations in the internal properties of clouds (e.g., virial parameter, Mach number, Alfvénic Mach number, or free-fall time) or large scale motions. We have demonstrated above that the distribution in the observed $\epsilon_{\mathrm{ff}}$ of Milky Way GMCs is far too broad to be explained by any of these models (equations 16, 20, 21, and 22).

A number of authors have suggested that the star formation rate on scales of GMCs and smaller increases systematically with time (see e.g., Palla \& Stahler 1999, Gutermuth et al. 2011 and Murray 2011 for observational evidence or see e.g., Lee et al. 2015, Murray \& Chang 2015, and Murray et al. 2015 for theoretical studies). Sampling clouds at different evolutionary stages with time-varying star formation rate may give rise to the broad distributions in $\epsilon$ and $\epsilon_{\mathrm{ff}}$. In this section, we allow $\epsilon_{\mathrm{ff}}$ to be time-dependent and compare the expected width in the distribution of $\epsilon_{\mathrm{ff}}$ to that observed. We note that the explicit time-dependence of $\epsilon_{\mathrm{ff}}$ arises from the time-varying turbulent structure (i.e., the size-linewidth relation itself changes with time due to the interplay between turbulence and gravity; see Goldbaum et al. 2011 and Murray \& Chang 2015). This explicit timedependence should not be confused with the implicit time-dependence portrayed by Hennebelle \& Chabrier (2011) whose model accounts for the re-assembly of turbulent structure that is static (i.e., the turbulent flows follow the same size-linewidth relation each time they are recycled).

We proceed to test the effect of explicit time evolution of $\epsilon_{\mathrm{ff}}$ on the distribution of $\epsilon_{\mathrm{ff}}$. First, we generalize the Krumholz \& McKee (2005) model to allow for timevariable $\epsilon_{\mathrm{ff}}$,

$$
\frac{d M_{*}}{d t}=\epsilon_{\mathrm{ff}, 0}\left(\frac{t}{\tau_{\mathrm{ff}}}\right)^{\delta} \frac{M_{g}}{\tau_{\mathrm{ff}}}
$$

where $t=0$ corresponds to the time at which the first star forms, and $\delta=0$ corresponds to the constant $\epsilon_{\mathrm{ff}}$ model.

Next, we use this prescription for $\epsilon_{\mathrm{ff}}$ in the model of Feldmann \& Gnedin (2011) which describes the concomitant evolution of the stellar mass and the gas mass of a star-forming cloud:

$$
\begin{aligned}
\frac{d M_{g}}{d t} & =-\epsilon_{\mathrm{ff}, 0}\left(\frac{t}{\tau_{\mathrm{ff}}}\right)^{\delta} \frac{M_{g}}{\tau_{\mathrm{ff}}}-\alpha M_{*}+\gamma, \\
\frac{d M_{*}}{d t} & =\epsilon_{\mathrm{ff}, 0}\left(\frac{t}{\tau_{\mathrm{ff}}}\right)^{\delta} \frac{M_{g}}{\tau_{\mathrm{ff}}} .
\end{aligned}
$$

The quantity $\alpha$ parametrizes the rate at which stellar feedback disrupts the host GMC, while $\gamma$ is the (possibly time dependent) rate of gas accretion onto the GMC.

Equations 24 and 25 are coupled ordinary differential equations (ODEs) whose solutions are $M_{g}(t)$ and $M_{\star}(t)$. Massive stars that contribute most to the ionizing radiation typically live for $\left\langle t_{\mathrm{ms}, \mathrm{q}}\right\rangle \simeq 3.9$ Myrs so we define the mass in these "live" stars as $M_{\star, \text { live }}(t)=M_{\star}(t)-$ $M_{\star}\left(t-\left\langle t_{\mathrm{ms}, \mathrm{q}}\right\rangle\right)(t)$. We can then write the star formation efficiency as $\epsilon(t) \equiv M_{\star, \text { live }}(t) /\left(M_{g}(t)+M_{\star, \text { live }}(t)\right)$ and the star formation rate per free fall time as $\eta_{\mathrm{ff}}(t) \equiv$ $\epsilon(t) \tau_{\mathrm{ff}} /\left\langle t_{\mathrm{ms}, \mathrm{q}}\right\rangle$. Note that $\eta_{\mathrm{ff}}$ is analogous to the observed $\epsilon_{\mathrm{ff}}$, not to be confused with $\epsilon_{\mathrm{ff}, 0}\left(t / \tau_{\mathrm{ff}}\right)^{\delta}$.

We illustrate the predicted evolution and the distribution of $\eta_{\mathrm{ff}}$ of this model in Figure 8 for three different $\delta=0,1,2$ assuming no accretion $(\gamma=0)$. We set $\tau_{\mathrm{ff}}$ to the median GMC free-fall time $\tau_{\mathrm{ff}} \sim 6.7 \mathrm{Myrs}$ and set $\alpha=3.5$ so that the cloud disperses in $\sim 20$ Myrs e.g., Williams \& McKee 1997 and Murray 2011. In Section 6.5 below we obtain a mean cloud lifetime using MML16 clouds of 21-24 Myrs. To isolate the effect of $\delta$, we assume all the host GMCs have the same $\alpha_{\text {vir }}$ and $\mathcal{M}$, or 

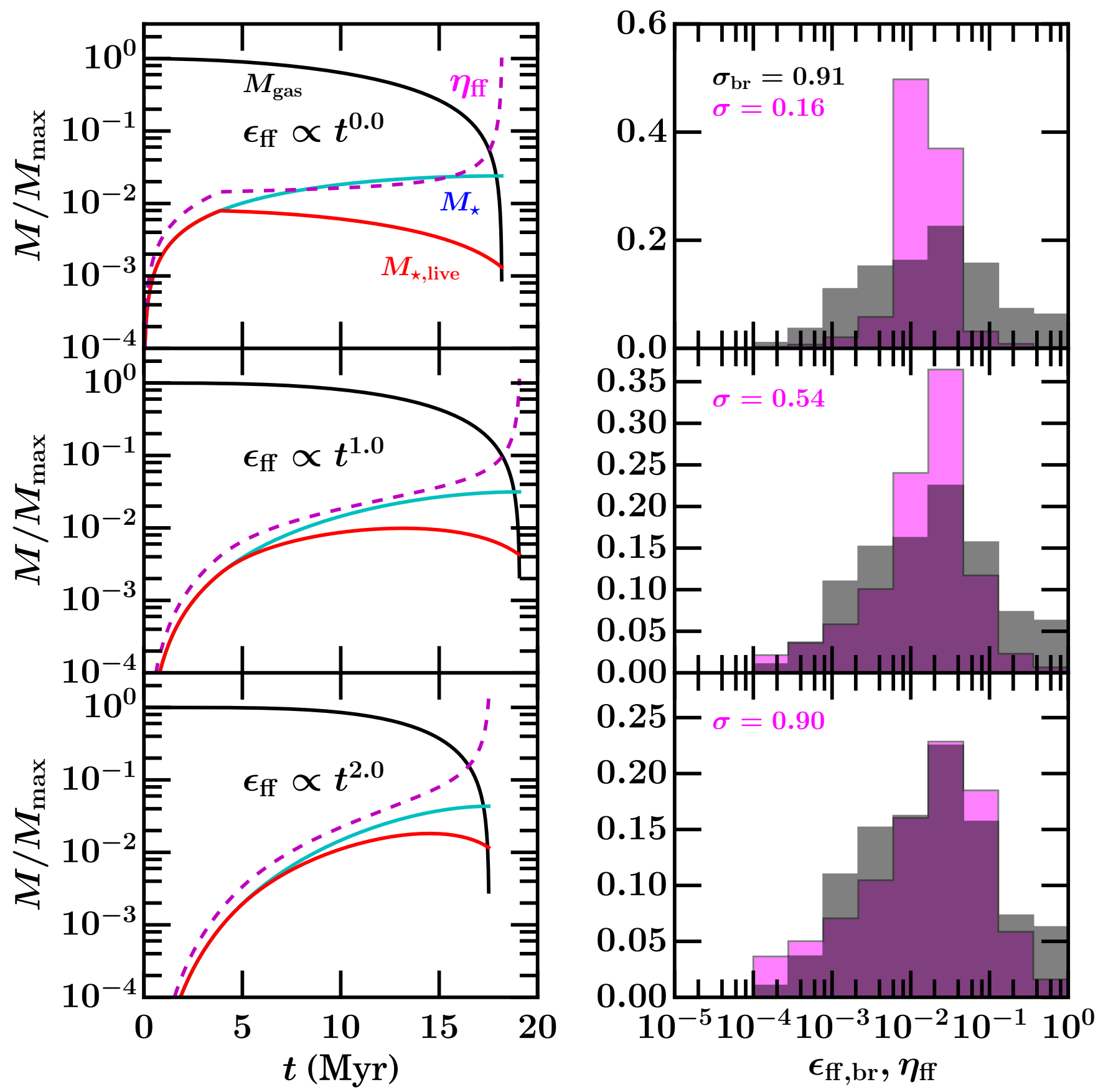

Figure 8. Left: the evolution of the GMC gas mass $M_{g}$ (black line), stellar mass $M_{\star}$ (cyan line), and live stellar mass $M_{\star, \text { live }}$ (red line; those with effective lifetime of $\left\langle t_{\mathrm{ms}, \mathrm{q}}\right\rangle \sim 4$ Myrs), together with the star formation rate per free-fall time $\eta_{\mathrm{ff}} \equiv\left[M_{\star, \text { live }} /\left(M_{g}+M_{\star, \text { live }}\right)\right]\left(\tau_{\mathrm{ff}} /\left\langle t_{\mathrm{ms}, \mathrm{q}}\right\rangle\right)$ (magenta dashed line) for three different values of $\delta=0,1,2$ in the model described by equations 24 and 25 . We use the observed median free-fall time $\tau_{\mathrm{ff}} \simeq 6.7$ Myrs . Right: the predicted distributions of $\eta_{\mathrm{ff}}$ (magenta histograms) together with the observed distributions (gray histograms). We assume no gas accretion (results for constant accretion models are similar). A quadratic growth $\epsilon_{\mathrm{ff}} \propto t^{2}$ (bottom panels) reproduces the observed scatter in $\epsilon_{\mathrm{ff}}$ the best. 
more precisely, the same intrinsic value of $\epsilon_{\mathrm{ff}, 0}$. We account for variations in these parameters after integrating the ODEs by adding the variations in quadrature.

The distribution of $\eta_{\mathrm{ff}}$ that we calculate from the model with $\delta=0$ (the constant $\epsilon_{\mathrm{ff}}$ model) features a very sharp peak. Because the lifetime of GMCs ( $\sim 20$ Myrs $)$ is substantially longer than the lifetime of live stars $(\sim 4$ Myrs $)$, the distribution in $\eta_{\mathrm{ff}}$ is dominated by the period in which both the live stellar mass and the host GMC mass are roughly constant with $\epsilon \sim \epsilon_{\mathrm{ff}, 0}\left(4 \mathrm{Myrs} / \tau_{\mathrm{ff}}\right)$ and therefore a constant $\eta_{\mathrm{ff}} \sim \epsilon_{\mathrm{ff}, 0}$. The small scatter (0.16 dex) arises partly from the initial rise in the live stellar mass, and partly from the final rapid decline in GMC mass around $\sim 20$ Myrs, when the stellar feedback disrupts the host GMC.

To make a fair comparison to the observed scatter in $\epsilon_{\mathrm{ff}}$, we add in quadrature the scatter from the initial and final transients, $0.16 \mathrm{dex}$, and the dispersion in $\epsilon_{\mathrm{ff}, 0}=$ 0.24 dex, to find a total predicted dispersion of $\sigma_{\log } \eta_{\mathrm{ff}}=$ 0.29 . Compared to the observed $\sigma_{\mathrm{br}}=0.91 \pm 0.22 \mathrm{dex}$, the scatter predicted by the models of time-independent $\epsilon_{\mathrm{ff}}$ is too small by 2.8 standard deviations.

Allowing for a monotonic increase in $\epsilon_{\mathrm{ff}}$ with time significantly broadens the distribution in $\epsilon$ and $\epsilon_{\mathrm{ff}}$ (see second and third rows in Figure 8). Not only does the mass in live stars monotonically increase over most of the cloud lifetime, it also increases more gradually from time zero. When $\delta=1$ (as predicted by Lee et al. 2015 and Murray \& Chang 2015), we find $\sigma=0.54$. Adding this in quadrature to the scatter in GMC properties, we find a total dispersion of $\sigma_{\log \eta_{\mathrm{ff}}}=0.59$ (1.4-sigma away from $\left.\sigma_{\text {br }}\right)$. Similar calculations show a total dispersion of $\sigma_{\log \eta_{\mathrm{ff}}}=0.93$ for $\delta=2.0$ (within one standard deviation from $\left.\sigma_{\mathrm{br}}\right)$.

Models assuming constant star formation rate per free-fall time predict too narrow a distribution in $\epsilon_{\mathrm{ff}}$. Using the simple model of Feldmann \& Gnedin (2011), $\epsilon_{\mathrm{ff}} \propto t^{2}$ - equivalently, the stellar mass increases as $M_{*}(t) \sim t^{3}$-fits the data better. Our result demonstrates that a time-varying star formation rate is in better agreement with the data compared to timeindependent models. Determining the exact form of $\epsilon_{\mathrm{ff}}$ that best fits the observation will require a careful parameter study which is beyond the scope of this paper.

We note that all predicted distributions of $\eta_{\mathrm{ff}}$ (whether the star formation rate is assumed to be time-dependent or constant) feature a deficit towards the high end and an excess towards the low end compared to the observations. The simple model of Feldmann \& Gnedin (2011) assumes that feedback from stars begins to destroy the host GMC as soon as the stars form. If the feedback takes the form of gas pressure in HII regions, or of radiation pressure, the host GMC will not be significantly affected until either pressure (or their sum) overcomes the pressure associated with the self-gravity of the GMC. Altering the model to account for this threshold effect will extend the distribution of $\epsilon$ to lower values. We leave this and similar model building efforts to future work.

\subsection{Cloud Lifetimes}

We estimate the average cloud lifetime using the starforming clouds (191 SFC-GMC complexes) and nonstar-forming clouds that are massive enough - and gravitationally bound - to birth stars. We show that clouds indeed live substantially longer than $\sim 4$ Myrs. This separation of time scales is crucial if we are to use the width of the distribution of $\epsilon$ (or $\epsilon_{\mathrm{ff}}$ ) to distinguish between constant star formation rate models and models which allow for variations of the star formation rate with time.

Clouds that harbor at least one SFC are likely near the end of their lives: their SFCs have already carved out bubbles of size 10-100 pc. The lifetime of clouds can then be estimated by multiplying the effective lifetime of live stars by the ratio of the total number of potentially star-forming clouds to the number of clouds that have SFCs. We define potentially star-forming clouds as those that are both massive enough to birth Orion Nebula Cluster (i.e., $M_{\text {gas }} \geq M_{*} \sim 1000 M_{\odot}$ ) and gravitationally bound ( $\alpha_{\text {vir }}<3.3$; see Appendix of MML16); there are 1014 such clouds. The average cloud lifetime is then $((1014+191) / 191) \times 4$ Myrs $\sim 24$ Myrs. The lifetime reduces to $21 \mathrm{Myrs}$ if we place the cloud lower mass limit at $10^{4} M_{\odot}$ instead.

\section{THE MILKY WAY STAR FORMATION RATE SURFACE DENSITY PROFILE}

Kennicutt \& Evans (2012), in their Figure 7, show that the star formation rate per unit area $\dot{\Sigma}_{\star}$ falls off more slowly than the surface density of molecular gas $\Sigma_{\text {gas }}$. By contrast, in the nearby galaxy NGC 6946, $\dot{\Sigma}_{\star}$ is observed to follow $\Sigma_{\text {gas }}$ closely. The information on star formation rate used by Kennicutt \& Evans (2012) date back to Guesten \& Mezger (1982). The catalog of SFCs and MML16 clouds used in this paper should provide the most up-to-date and the most complete estimate of star formation rate and the molecular gas mass. Using the sample of star-forming clouds, we illustrate in Figure 9 how even in the Milky Way, $\dot{\Sigma}_{\star}$ tracks well $\Sigma_{\text {gas }}$ as a function of galactocentric radius. In particular, both the star formation rate and the gas density profiles are wellfitted with an exponential profile of scale length $\sim 2.0$. The trend observed in $\dot{\Sigma}_{\star}$ is anti-correlated with the profile of $\alpha_{\text {vir }}$ shown in Figure 14 of MML16: where $\alpha_{\text {vir }}$ rises, $\dot{\Sigma}_{\star} \operatorname{dips}$ (inside $4 \mathrm{kpc}$ and outside $8 \mathrm{kpc}$ ) and where $\alpha_{\text {vir }} \operatorname{dips}, \dot{\Sigma}_{\star}$ rises (between 4 and $8 \mathrm{kpc}$ ). This anti-correlation is expected since stars should form in 


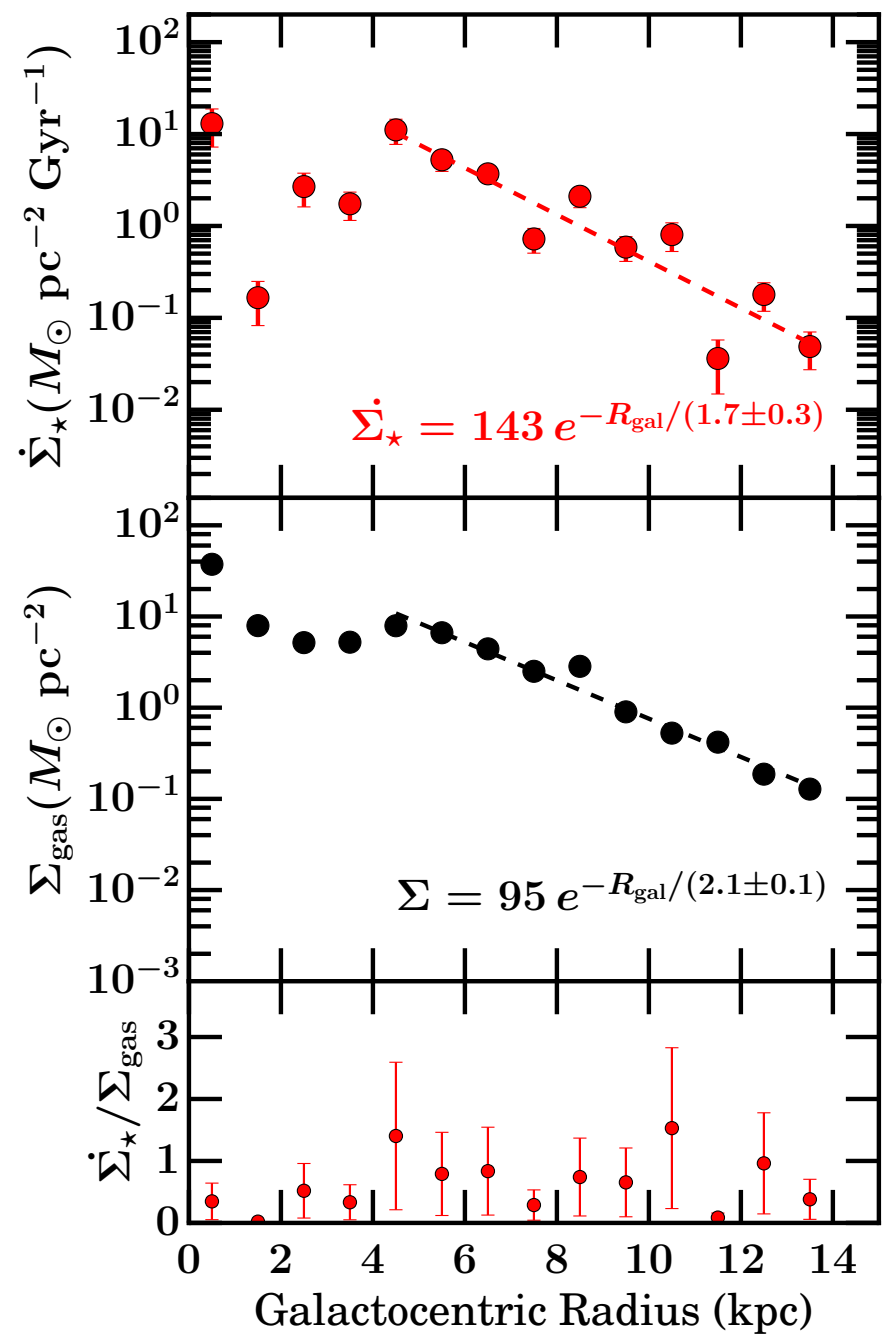

Figure 9. Top: star formation rate per unit area $\dot{\Sigma}_{\star}$ as a function of galactocentric radius. Middle: gas surface density profile $\Sigma_{\text {gas }}$. Bottom: the ratio between the two profiles $\dot{\Sigma}_{\star} / \Sigma_{\text {gas }}$. Star formation is observed to follow the gas: they all show similar scale radius. Both profiles show a break at $\sim 4 \mathrm{kpc}$, consistent with the presence of a molecular ring.

gravitationally-bound regions.

\section{DISCUSSION AND SUMMARY}

We have demonstrated in this paper the importance of the scatter in the distribution of $\epsilon_{\mathrm{ff}}$ and $\epsilon$. Any successful model of cloud-scale star formation should reproduce not only the mean star formation rate but also the large scatter about the median. The spread in $\epsilon$ has long been known to be enormous: Mooney \& Solomon (1988), Scoville \& Good (1989), and Mead et al. (1990) find a minimum-maximum range of three orders of magnitude in $\epsilon$ as probed by FIR luminosity in a small sample of nearby molecular clouds. More recently, Lada et al. (2010) and Heiderman et al. (2010) count young stellar objects in a sample of nearby clouds, and show that the values of $\epsilon_{\mathrm{ff}}$ range up to a factor of ten larger than the nominal value of 0.02. Evans et al. (2014) report approximately an order of magnitude spread in $\epsilon_{\mathrm{ff}}$ both above and below 0.01 in nearby molecular clouds from the c2d and Gould's belt survey. Using dense clumps from ATLASGAL, Heyer et al. (2016) find $\epsilon_{\mathrm{ff}}$ can be as low as $\sim 0.001$.

We combined the Milky Way all-sky catalog of GMCs by M-A., Miville-Deschênes et al. (2016) and an all-sky catalog of SFCs by Lee et al. (2012) to build a large collection of star-forming clouds (see Section 2). Our MML16 catalog of GMCs contains 5469 valid clouds, which is about an order of magnitude more clouds than all previously published catalogs. Using the statistical power afforded by this catalog, we showed that both $\epsilon$ and $\epsilon_{\mathrm{ff}}$ of star-forming GMCs in the Milky Way have large dispersions: $\sigma_{\log \epsilon},=0.79 \pm 0.22 \mathrm{dex}$, and $\sigma_{\log \epsilon_{\mathrm{ff}}}=$ $0.91 \pm 0.22 \mathrm{dex}$, (see Sections 4 and 5 ). These results confirm and extend earlier observations. The error in the scatter is dominated by the statistical variations in the IMF (see Section 3).

Variations in internal cloud properties or their large scale motions cannot account for the large scatter in $\epsilon_{\mathrm{ff}}$. We rule out the constant star-formation rate model of Krumholz \& McKee (2005) since cloud-to-cloud variations in the virial parameter $\alpha_{\text {vir }}$ or the Mach number $\mathcal{M}$ do not produce a large enough scatter in $\epsilon_{\mathrm{ff}}$ (see Section 6.1). Even the improved model of turbulence-regulated star formation by Hennebelle \& Chabrier (2011) — that has a strong dependence on the Mach number - cannot account for the observed scatter. Similarly, we find at least an order of magnitude scatter in star formation rate about the collision-induced star formation law proposed by Tan (2000); the rate at which gas is converted to star on a cloud-to-cloud collisional timescale cannot be a constant (see Section 6.2). We see some evidence of decreasing $\epsilon_{\mathrm{ff}}$ with larger $\tau_{\mathrm{ff}} / \tau_{\text {dyn }}$ as expected by Padoan et al. (2012) but the large scatter for a given time ratio cannot be explained by varying magnetic field intensities (see Section 6.3).

One way to produce a large dispersion in both $\epsilon$ and $\epsilon_{\mathrm{ff}}$ is to arrange for a time-variable rate of star formation in a GMC with fixed gas mass, density, and velocity dispersion (see Section 6.4). Generalizing the turbulenceregulated star formation model of Krumholz \& McKee (2005) to allow time-variable $\epsilon_{\mathrm{ff}}$ in the prescription of Feldmann \& Gnedin (2011), we find that $\epsilon_{\mathrm{ff}} \propto t^{2}$ is most consistent with our data. We conclude that star formation is dynamic on the GMC-scale.

Our study concerns the properties of star formation in clouds that have already formed or in the process

\footnotetext{
5 The time-varying $\epsilon_{\mathrm{ff}}$ may arise from the evolution of starforming clumps embedded in each GMC.
} 
of forming stars. By construction, we have limited our analysis to 191 SFC-GMC complexes out of 5469. We found 1014 clouds unmatched to SFCs that have the potential to form star clusters (see Section 6.5). We surmise that most $(5469-1014-191=4264 ; 78 \%$ by number) of the GMCs are gravitationally unbound and will never form massive star clusters.

It is possible that some locally dense clumps within an unbound GMC form stars (see e.g., Dobbs et al. 2011). These clumps likely give birth to lower mass star clusters that our star formation tracers are not sensitive to. Throughout our analysis, we used free-free emission as a proxy for stellar mass. A more direct measurement would be to count young stellar objects (YSOs). For known massive clusters, we find a generally good agreement in the measured stellar mass between freefree emission and YSO counts (see Figure 2). A more complete comparison may be possible using all-sky YSO catalogs (e.g., Marton et al. 2016). This is an important and natural avenue for future improvement.

\subsection{Comments on Stellar Feedback}

If the rate of star formation in the most massive $\left(10^{6} M_{\odot}\right.$ and higher $)$ GMCs does accelerate, the process of star formation must halt before $\epsilon \gtrsim 0.1$, roughly the largest value we see in such clouds. Stellar feedback -in the form of stellar winds, radiation pressure, protostellar jets, and supernovae - from massive star clusters can disrupt the natal cloud and inhibit future star formation. The idea that stellar feedback destroys GMCs was proposed long ago by Larson (1981). Galactic-scale numerical simulations (e.g., Hopkins et al. 2011 and FaucherGiguère et al. 2013) find that the effects of stellar feedback are necessary for regulating star formation rates to the Kennicutt-Schmidt value. Turbulence in the interstellar medium alone cannot slow the rate of star formation.

The existence of large expanding bubbles - a few to $\sim 100$ parsecs wide - associated with regions bright in free-free emission is evidence for the effects of stellar feedback from young clusters (referred to as SFCs by
Rahman \& Murray 2010 and Lee et al. 2012). These massive young clusters, which power most of the freefree emission in the Milky Way, are identified by clear cavities in the $8 \mu \mathrm{m}$ emission. The clusters are found in massive GMCs, but the star clusters are not enshrouded in molecular gas - they are instead in regions of ionized gas which are enshrouded in molecular gas. In some cases, the massive stars may still be accreting gas from their natal protostellar disks but the disks are not accreting gas from the host GMC.

We have examined our sample to see if the feedback from stars is affecting their host GMCs globally. There was no clear correlation between the variations in $\alpha_{\text {vir }}$ and the ratio of disruptive forces (gas and radiation pressure) to the binding force (dynamical pressure) of the host GMC. We also found no clear correlation between the size of the bubbles blown by SFCs in the host clouds and $\alpha_{\text {vir }}$. It may be that the effects of radiation and gas pressure will only become evident later in the evolutionary history of the GMC, or it may be that other forms of feedback (e.g., supernovae) are responsible for disrupting the clouds. Given that GMCs in the Milky Way are found near spiral arms, combined with our finding that the a large fraction of $10^{6} M_{\odot}$ GMCs are most likely gravitationally bound $\left(\alpha_{\text {vir }}<1\right)$, it seems unlikely that they simply disperse on their own.

The creation of the MML16 catalog of GMCs is made possible by the all-sky CO data provided by T. Dame. We thank the anonymous referee for their constructive feedback that helped to improve this paper significantly. We thank L. Blitz, G. Chabrier, E. Chiang, C-A. Faucher-Giguére, C. Federrath, R. Feldmann, N. Gnedin, P. Hopkins, M. Krumholz, P-S. Li, C. Matzner, C. Mckee, E. Ostriker, E. Quataert, S. Stahler, J. Tan, and E. Vazquez-Semadeni for valuable discussions. We also thank H. Isaacson, R. Trainor, and J. Wang for their advice in statistical analyses. EJL is supported in part by NSERC of Canada under PGS D3 and the Berkeley fellowship. NWM is supported in part by NSERC of Canada. This research was undertaken, in part, thanks to funding from the Canada Research Chairs program.

\section{REFERENCES}

Anderson, L. D., Zavagno, A., Rodón, J. A., et al. 2010, A\&A, 518, L99

Bica, E., Dutra, C. M., Soares, J., \& Barbuy, B. 2003, A\&A, 404, 223

Bolatto, A. D., Wolfire, M., \& Leroy, A. K. 2013, ARAA, 51, 207

Brand, J., \& Blitz, L. 1993, A\&A, 275, 67

Caselli, P., \& Myers, P. C. 1995, ApJ, 446, 665

Chabrier, G., Hennebelle, P., \& Charlot, S. 2014, ApJ, 796, 75

Cho, W., \& Kim, J. 2011, MNRAS, 410, L8

Clemens, D. P. 1985, ApJ, 295, 422
Collins, D. C., Kritsuk, A. G., Padoan, P., et al. 2012, ApJ, 750, 13

Crutcher, R. M. 2012, ARA\&A, 50, 29

Da Rio, N., Robberto, M., Hillenbrand, L. A., Henning, T., \&

Stassun, K. G. 2012, ApJ, 748, 14

Dame, T. M., Elmegreen, B. G., Cohen, R. S., \& Thaddeus, P. 1986, ApJ, 305, 892

Dame, T. M., Hartmann, D., \& Thaddeus, P. 2001, ApJ, 547, 792 Dib, S. 2014, MNRAS, 444, 1957 
Dobbs, C. L., Burkert, A., \& Pringle, J. E. 2011, MNRAS, 413, 2935

Evans, II, N. J., Dunham, M. M., Jørgensen, J. K., et al. 2009, ApJS, 181, 321

Evans, II, N. J., Heiderman, A., \& Vutisalchavakul, N. 2014, ApJ, 782, 114

Faucher-Giguère, C.-A., Quataert, E., \& Hopkins, P. F. 2013, MNRAS, 433, 1970

Federrath, C. 2013, MNRAS, 436, 3167

Federrath, C., \& Klessen, R. S. 2012, ApJ, 761, 156

—. 2013, ApJ, 763, 51

Feldmann, R., \& Gnedin, N. Y. 2011, ApJL, 727, L12

Fich, M., Blitz, L., \& Stark, A. A. 1989, ApJ, 342, 272

García, P., Bronfman, L., Nyman, L.-Å., Dame, T. M., \& Luna, A. 2014, ApJS, 212, 2

Goldbaum, N. J., Krumholz, M. R., Matzner, C. D., \& McKee, C. F. 2011, ApJ, 738, 101

Grabelsky, D. A., Cohen, R. S., Bronfman, L., \& Thaddeus, P. 1988, ApJ, 331, 181

Guesten, R., \& Mezger, P. G. 1982, Vistas in Astronomy, 26, 159

Gutermuth, R. A., Pipher, J. L., Megeath, S. T., et al. 2011, ApJ, 739, 84

Heiderman, A., Evans, II, N. J., Allen, L. E., Huard, T., \& Heyer, M. 2010, ApJ, 723, 1019

Hennebelle, P., \& Chabrier, G. 2011, ApJL, 743, L29

—. 2013, ApJ, 770, 150

Hennebelle, P., \& Falgarone, E. 2012, Astronomy and Astrophysics Review, 20, 55

Heyer, M., Gutermuth, R., Urquhart, J. S., et al. 2016, A\&A, 588, A29

Heyer, M., Krawczyk, C., Duval, J., \& Jackson, J. M. 2009, ApJ, 699, 1092

Homeier, N. L., \& Alves, J. 2005, A\&A, 430, 481

Hopkins, P. F., Kereš, D., Oñorbe, J., et al. 2014, MNRAS, 445, 581

Hopkins, P. F., Quataert, E., \& Murray, N. 2011, MNRAS, 417, 950

Jackson, J. M., Rathborne, J. M., Shah, R. Y., et al. 2006, ApJS, 163,145

Kennicutt, R. C., \& Evans, N. J. 2012, ARA\&A, 50, 531

Kennicutt, Jr., R. C. 1989, ApJ, 344, 685

—. 1998, ApJ, 498, 541

Koenig, X. P., Allen, L. E., Gutermuth, R. A., et al. 2008, ApJ, 688,1142

Kritsuk, A. G., Norman, M. L., \& Wagner, R. 2011, ApJL, 727, L20

Krumholz, M. R., Dekel, A., \& McKee, C. F. 2012, ApJ, 745, 69

Krumholz, M. R., Fumagalli, M., da Silva, R. L., Rendahl, T., \& Parra, J. 2015, MNRAS, 452, 1447

Krumholz, M. R., \& McKee, C. F. 2005, ApJ, 630, 250

Krumholz, M. R., \& Tan, J. C. 2007, ApJ, 654, 304
Lada, C. J., Lombardi, M., \& Alves, J. F. 2010, ApJ, 724, 687

Larson, R. B. 1981, MNRAS, 194, 809

Lee, E. J., Chang, P., \& Murray, N. 2015, ApJ, 800, 49

Lee, E. J., Murray, N., \& Rahman, M. 2012, ApJ, 752, 146

Mallick, K. K., Kumar, M. S. N., Ojha, D. K., et al. 2013, ApJ, 779,113

Marton, G., Tóth, L. V., Paladini, R., et al. 2016, MNRAS, 458, 3479

McKee, C. F., \& Tan, J. C. 2003, ApJ, 585, 850

McKee, C. F., \& Williams, J. P. 1997, ApJ, 476, 144

McLaughlin, D. E., \& Pudritz, R. E. 1997, ApJ, 476, 750

Mead, K. N., Kutner, M. L., \& Evans, II, N. J. 1990, ApJ, 354, 492

Mooney, T. J., \& Solomon, P. M. 1988, ApJL, 334, L51

Morales, E. F. E., Wyrowski, F., Schuller, F., \& Menten, K. M. 2013, A\&A, 560, A76

Murray, D. W., Chang, P., Murray, N. W., \& Pittman, J. 2015, ArXiv e-prints, arXiv:1509.05910

Murray, N. 2011, ApJ, 729, 133

Murray, N., \& Chang, P. 2015, ApJ, 804, 44

Murray, N., \& Rahman, M. 2010, ApJ, 709, 424

Myers, P. C., \& Fuller, G. A. 1992, ApJ, 396, 631

Oppenheimer, B. D., Davé, R., Kereš, D., et al. 2010, MNRAS, 406, 2325

Ostriker, E. C., \& Shetty, R. 2011, ApJ, 731, 41

Padoan, P., Haugbølle, T., \& Nordlund, Å. 2012, ApJL, 759, L27

Padoan, P., \& Nordlund, Å. 2011, ApJ, 730, 40

Palla, F., \& Stahler, S. W. 1999, ApJ, 525, 772

Plume, R., Jaffe, D. T., Evans, II, N. J., Martín-Pintado, J., \& Gómez-González, J. 1997, ApJ, 476, 730

Portegies Zwart, S. F., McMillan, S. L. W., \& Gieles, M. 2010, ARA\&A, 48, 431

Rahman, M., \& Murray, N. 2010, ApJ, 719, 1104

Rosolowsky, E. W., Pineda, J. E., Kauffmann, J., \& Goodman, A. A. 2008, ApJ, 679,1338

Schwartz, C. M., Martin, C. L., Chandar, R., et al. 2006, ApJ, 646,858

Scoville, N. Z., \& Good, J. C. 1989, ApJ, 339, 149

Solomon, P. M., Rivolo, A. R., Barrett, J., \& Yahil, A. 1987, ApJ, 319, 730

Suwannajak, C., Tan, J. C., \& Leroy, A. K. 2014, ApJ, 787, 68

Tan, J. C. 2000, ApJ, 536, 173

Thompson, T. A., Quataert, E., \& Murray, N. 2005, ApJ, 630, 167

Vázquez-Semadeni, E., González, R. F., Ballesteros-Paredes, J., Gazol, A., \& Kim, J. 2008, MNRAS, 390, 769

Williams, J. P., de Geus, E. J., \& Blitz, L. 1994, ApJ, 428, 693

Williams, J. P., \& McKee, C. F. 1997, ApJ, 476, 166

Wolk, S. J., Broos, P. S., Getman, K. V., et al. 2011, ApJS, 194, 12 